\title{
Probing the pinning landscape in type-II superconductors via Campbell penetration depth
}

\author{
R. Willa, ${ }^{1}$ V.B. Geshkenbein, ${ }^{1}$ and G. Blatter ${ }^{1}$ \\ ${ }^{1}$ Institute for Theoretical Physics, ETH Zurich, 8093 Zurich, Switzerland
}

(Dated: September 7, 2021)

\begin{abstract}
Type-II superconductors owe their magnetic and transport properties to vortex pinning, the immobilization of flux quanta through material inhomogeneities or defects. Characterizing the potential energy landscape for vortices, the pinning landscape (or short, pinscape), is of great technological importance. Besides measurement of the critical current density $j_{c}$ and of creep rates $S$, the $a c$ magnetic response provides valuable information on the pinscape which is different from that obtained through $j_{c}$ or $S$, with the Campbell penetration depth $\lambda_{\mathrm{C}}$ defining a characteristic quantity well accessible in an experiment. Here, we derive a microscopic expression for the Campbell penetration depth $\lambda_{\mathrm{C}}$ using strong pinning theory. Our results explain the dependence of $\lambda_{\mathrm{C}}$ on the state preparation of the vortex system and the appearance of hysteretic response. Analyzing different pinning models, metallic or insulating inclusions as well as $\delta T_{c^{-}}$and $\delta \ell$-pinning, we discuss the behavior of the Campbell length for different vortex state preparations within the phenomenological $H-T$ phase diagram and compare our results with recent experiments.
\end{abstract}

\section{INTRODUCTION}

In a type-II superconductor, the magnetic field $H$ penetrates the material in the form of vortices ${ }^{12}$, individually capturing a superconducting flux quantum $\Phi_{0}=$ $h c / 2 e$ and together forming a triangular Abrikosov lattice generating the magnetic induction $B$ inside the sample. In ideal superconductors, an applied current density $j$ generates a Lorentz force $F_{\mathrm{L}}=j B / c$, setting the vortex lattice in motion. The resulting velocity $v$ produces an electric field $E=v B / c$ which renders the current transport dissipative $e^{3}$. The material's response then is characterized by the flux-flow resistivity $\rho_{\mathrm{ff}} \simeq \rho_{n} B / H_{c 2}$, with $\rho_{n}$ the normal state resistivity and $H_{c 2}$ the upper critical field. In real materials, chemical impurities or crystallographic defects immobilize vortices, thereby restoring dissipation-free electric transport for currents $j$ below a critical value $j_{c}$. Achieving large critical currents $j_{c}$ is a prime task in optimizing superconductors for technological applications. Recently, analytic, numerical, and experimental studies have been used in a concerted effort to investigate the fundamental mechanisms governing vortex pinning 4 . Such a program relies on a proper characterization of the material's pinning landscape or pinscape. Besides measurement of the critical current density $j_{c}$, the analysis of the material's ac magnetic response ${ }^{6 / 7}$ as quantified through the Campbell penetration depth $\lambda_{\mathrm{C}}$ provides valuable infomation on the bulk pinning parameters. In this paper, we present a microscopic foundation for the Campbell penetration length $\lambda_{\mathrm{C}}$ which allows to connect the result of $a c$ magnetic response measurements to microscopic parameters of the pinscape.

When measuring a material's $a c$ response, a small magnetic field $h_{a c}$ is applied on top of a large $d c$ field $B_{0}$. In Campbell's original phenomenological approach $\frac{677}{\text {, the }}$ $a c$ field forces vortices to oscillate within their pinning potentials which are conveniently characterized by an ef- fective harmonic potential well $\alpha u^{2} / 2$, with $u$ denoting the vortex displacement. A measurement of the Campbell length $\lambda_{\mathrm{C}} \propto \alpha^{-1 / 2}$ then informs about the curvature of this 'single-vortex' potential. Later, the $a c$ magnetic response has been further discussed in the context toy model $\$ \frac{819}{}$ assuming a piecewise parabolic potentials. In the wake of the discovery of high-temperature superconductivity ${ }^{10}$, the frequency response of the vortex state has attracted renewed attention, especially in the context of vortex creep 11 15. Recent experimental developments in the field have been reviewed in Refs. [16] and 17.

A phenomenological approach as described above cannot relate the measured penetration depth $\lambda_{\mathrm{C}}$ to the microscopic parameters of the pinscape. In particular, it is unclear how such a simple description can deal with the Bean critical state ${ }^{18}$. The latter is realized at $j=j_{c}$ where the pinning landscape acts with its maximal force $F_{c}$ against the Lorentz force $F_{\mathrm{L}}$ and establishes a self-organized critical state resembling a sandpile ${ }^{19}$, with avalanche-type motion of vortices triggered upon increasing the magnetic field. The phenomenological mode ${ }^{17}$ describes this situation by a vanishing curvature $\alpha\left(j \rightarrow j_{c}\right) \rightarrow 0$, resulting in a formally diverging Campbell length and hence a full penetration of the $a c$ signal. Such a divergent signature has not been observed in experiments ${ }^{17}$; rather, it has been found that the Campbell length can even decrease when going from a fieldcooled state (FC) to a Bean critical (or zero-field-cooled, ZFC) $\operatorname{state}^{20}$.

Vortex pinning, including $j_{c}$, is usually described within either of two frameworks, weak collective pinning due to the joint action of many weak defects or strong pinning produced by a low density of strong impurities 21122]. Within our microscopic description, we make use of strong pinning theory and relate the measured penetration depth $\lambda_{\mathrm{C}}$ to microscopic parameters of the pinscape. Most interestingly, it turns out that $j_{c}$ and $\lambda_{\mathrm{C}}$ are determined by different microscopic parameters: 
while the critical current density $j_{c}$ involves the characteristic jump in energy of strong pinning theory, the Campbell length involves the jump in the pinning force. The scaling $j_{c} \sim c \xi B / \lambda_{\mathrm{C}}^{2}$ used in the past then picks up a non-trivial dependence on the strong pinning (or Labusch) parameter $\kappa>1$, e.g., $j_{c} \sim\left(c \xi B / \lambda_{\mathrm{C}}^{2}\right)(\kappa-1)^{3 / 2}$ at the onset of strong pinning when $\kappa-1 \ll 1$ and $j_{c} \sim\left(c \xi B / \lambda_{\mathrm{C}}^{2}\right) \kappa$ for very strong pinning $\kappa \gg 1$. The quantitative power of strong pinning theory provides further interesting results such as the dependence of $\lambda_{\mathrm{C}}$ on the vortex state (e.g., field-cooled versus zero-fieldcooled) or the appearance of hysteretic behavior upon temperature cycling. Furthermore, mapping out the behavior of $\lambda_{\mathrm{C}}$ within the $H$-T phase diagram allows to draw interesting conclusions on the character of the pinning centers. While our analysis focuses on bulk characteristic parameters of the pinscape, different types of scanning techniques have been used recently to obtain a direct microscopic image of the potential landscape seen by individual vortices $\$ 2324$.

In the following, we first review the general approach to the $a c$ response (Sec. II) and then introduce the strong pinning formalism in Sec. III. We derive a quantitative relation between the Campbell length and the microscopic pinning potential and discuss the generic dependence of $\lambda_{\mathrm{C}}$ on the state preparation (e.g., FC vs. ZFC) as well as hysteretic effects. In Sec. [IV] we analyze the pinning characteristics of four types of defects, namely insulating and metallic inclusions as well as $\delta T_{c^{-}}$and $\delta \ell$-pins. In Sec. $\mathrm{V}$ we compare our findings with recent measurements20 on $\mathrm{SrPd}_{2} \mathrm{Ge}_{2}$ and find good overall agreement using a pinscape with a scaling characteristic of insulating defects. A brief account of parts of this work can be found in Refs. 25] and 26.

\section{II. ac MAGNETIC RESPONSE}

We analyze the magnetic response of a bulk superconducting sample subject to a static field $H$ and a parallel $a c$ perturbation with a small amplitude $h_{a c} \ll H$. While the magnetic field $H$ induces a vortex lattice with an average induction $B_{0}$ in the sample, the small $a c$-field induces motion of these vortices. We choose a geometry with a superconductor filling the half-space $X>0$ with the sample surface, magnetic field, and $Z$-axis arranged in parallel. This corresponds, up to finite size effects, to a sample in a slab geometry arranged parallel to $Z$ where demagnetization effects are absent. We will briefly discuss the geometry of a thin platelet-like sample (arranged in the $X Y$-plane) at the end of the section.

On the macroscopic level, the vortex lattice can be described as an elastic medium and its response to the ac perturbation is reflected in a macroscopic displacement field $U(X, t)$ of the flux-lines. We use capital letters when describing macroscopic coordinates and displacements and denote their microscopic counterparts (below) by lower-case symbols. Starting from an initial field
$B_{0}(X)$ and current $j_{0}(X)$ at time $t=0$, the vector potential $\delta A=U(X, t) B_{0}(X)$ associated with the vortex lattice displacement $U$ induces time-dependent corrections of the form

$$
\begin{aligned}
\delta B(X, t) & =-B_{0}(0) \partial_{X} U(X, t), \\
\delta j(X, t) & =\frac{c}{4 \pi} B_{0}(0) \partial_{X}^{2} U(X, t) .
\end{aligned}
$$

The above expressions are valid in the linear-response regime where $U$ is the smallest length and $\left(\partial_{X} B_{0}\right) U \ll$ $B_{0}\left(\partial_{X} U\right)$. Integrating Eq. (1) over $X$, we find the flux $\phi(t)$ (per unit of length along $y$ ) that has penetrated the surface,

$$
\phi(t)=\int_{0}^{\infty} d X \delta B(X, t)=B_{0} U(0, t) .
$$

The distribution of this flux within the sample generates an additional induction $\delta B(X, t)$ on top of the $d c$ field $B_{0}(X)$, see also Sec. IIIC

Having reduced the change in fields and currents to the macroscopic displacement field $U(X, t)$ of vortices, we can find the dynamical response of $B(X, t)$ through the equation of motion of the flux-line lattice,

$$
\eta \dot{U}=F_{\mathrm{L}}(j, U)+F_{\text {pin }}(X, U),
$$

which balances the dissipative Bardeen-Stephen term with $\eta=B H_{c 2} / \rho_{n} c^{2}$ against the sum of Lorentz and pinning force densities, $F_{\mathrm{L}}=\left(j_{0}+\delta j\right) B_{0} / c$ and $F_{\text {pin }}=$ $F_{0}+\delta F_{\text {pin }}$. The static initial state is characterized by a pinning force $F_{0}$ that exactly compensates the Lorentz force $j_{0} B_{0} / c$ and the right-hand side vanishes identically (for a field-cooled sample both $F_{0}$ and $j_{0}$ vanish individually). Hence, the dynamic equation (4) assumes the form

$$
\eta \dot{U}-\frac{B_{0}^{2}}{4 \pi} \partial_{X}^{2} U-\delta F_{\operatorname{pin}}(X, U)=0 .
$$

Making use of Eq. (1), the external drive $\delta B(X=$ $0, t)=h_{a c} e^{-i \omega t}$ determines the boundary condition $\partial_{X} U(X, t)=-\left(h_{a c} / B_{0}\right) e^{-i \omega t}$. It remains to find an expression for the change in pinning force density $\delta F_{\text {pin }}(U)$.

Referring to Campbell's original work ${ }^{[6 / 7}$, one usually assumes that vortices oscillate reversibly in an effective parabolic pinning potential $\alpha U^{2} / 2$, what results in the phenomenological pinning force density

$$
\delta F_{\text {pin }}(U)=-\alpha U .
$$

Using this Ansatz, the equation of motion (5) can readily be solved for the displacement field $U(X, t)$, from which the field and current dynamics follow via Eqs. (1) and (2). One finds that the field oscillations

$$
\delta B(X, t)=h_{a c} e^{-X / \lambda_{\mathrm{C}}} e^{-i \omega t}
$$

decay into the sample with the characteristic length $\lambda_{\mathrm{C}}(\omega)$, which reduces to the Campbell length

$$
\lambda_{\mathrm{C}}=\left(\frac{B_{0}^{2}}{4 \pi \alpha}\right)^{1 / 2}
$$


at low frequencies $\omega \rightarrow 0$. The Campbell length thus relates the $a c$ penetration depth with the pinning properties of vortices through the curvature $\alpha$ of the effective pinning potential, a relation that has widely been used to characterize the pinning landscape.

However, inferring the properties of the pinning landscape from the experimental measurements based on a simple phenomenological model is prone to misjudgments. We therefore proceed with a microscopic approach based on strong pinning theory 2127 in order to determine the macroscopic response of the vortex state. Thereby the macroscopic equation of motion Eq. (5) can equally well be obtained from a microscopic route by averaging the equation of motion of individual vortices over an area much larger than $a_{0}^{2}$, with $a_{0}$ the inter-vortex distance. The evaluation of the change in pinning force $\delta F_{\text {pin }}(X, U)$, involves a proper average of the microscopic action of single pinning centers, a task we address in the following.

Before doing so, we briefly touch upon geometric aspects of the problem. For the slab geometry chosen here (with magnetic fields along $Z$ and currents along $Y$ ), the contributions from shear and tilt deformations average to zero and only the bulk compression modulus $c_{11}(\boldsymbol{k}=0)=B_{0}^{2} / 4 \pi$ enters in Eq. (5). In the platelet geometry, as opposed to the slab geometry, the field is arranged perpendicular to the largest sample dimension and demagnetization effects change both the size and effective direction of the $a c$ field component. For a sample thicker than $2 \lambda_{\mathrm{C}}$, the $a c$-component is screened and the effective drive $h_{a c}^{\text {eff }}$ is enhanced by $(w / d)^{1 / 2}$ and redirected parallel to the surface (here, $w$ and $d$ denote the width and thickness of the sample), see e.g., Refs. [28] and [29. The penetration of the $a c$-field then corresponds to tilting the vortices within a depth $\lambda_{\mathrm{C}}$ away from the surface and the relevant distortion modulus appearing in Eq. (5) is the bulk tilt modulus $c_{44}(\boldsymbol{k}=0)=B_{0}^{2} / 4 \pi$.

\section{STRONG PINNING}

\section{A. Formalism}

Within strong pinning theory as originally discussed by Labusch $^{27}$ and later by Larkin and Ovchinnikov 21 , a low density $n_{p}$ of pinning sites produces a finite pinning force by inducing large plastic deformations on the pinned vortices. We consider a lattice of vortices (directed along $z)$ with equilibrium coordinates $\boldsymbol{r}_{\mu}=\left(x_{\mu}, y_{\mu}\right)$ and an isolated defect at the origin defined through its pinning potential $e_{p}(\boldsymbol{r}, z) \simeq e_{p}(\boldsymbol{r}) \delta(z)$, with $\boldsymbol{r}=(x, y)$; as pins act independently, the action of a finite density of pins is trivially summed over. The interaction of the pin with the vortex lattice gains the system a local energy density

$$
\varepsilon_{p}(\boldsymbol{r}, z ; \boldsymbol{u})=\sum_{\mu} e_{p}(\boldsymbol{r}) \delta(z) \delta^{2}\left\{\boldsymbol{r}-\left[\boldsymbol{r}_{\mu}+\boldsymbol{u}\left(\boldsymbol{r}_{\mu}, z\right)\right]\right\}
$$

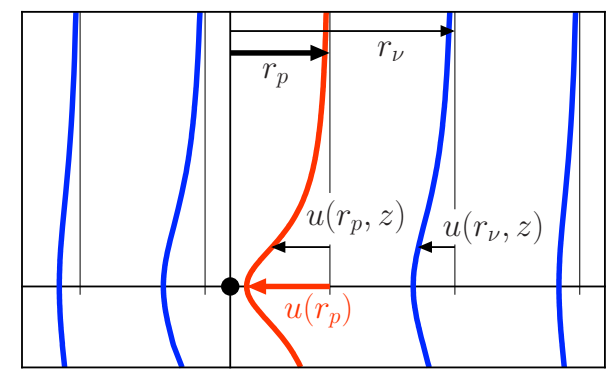

FIG. 1. Schematic view of the vortex distortion near a pinning center. The pinned vortex (red) with asymptotic position $\boldsymbol{r}_{\mu}=\boldsymbol{r}_{p}$ is deformed to $\boldsymbol{r}_{p}+\boldsymbol{u}\left(\boldsymbol{r}_{p}, z\right)$ by the presence of the pin (black dot). Nearby vortices with $\nu \neq \mu$ (blue) are deformed as well with their deformation $\boldsymbol{u}\left(\boldsymbol{r}_{\nu}, z\right)$ transported by the elastic Green's function $G\left(\boldsymbol{r}_{\nu}-\boldsymbol{r}_{p}, z_{\nu}-z\right)$. The full pinning problem can be reduced to a self-consistency equation for the deformation $\boldsymbol{u}\left(\boldsymbol{r}_{p}\right) \equiv \boldsymbol{u}\left(\boldsymbol{r}_{p}, 0\right)$ of the pinned vortex at the height $z=0$ of the defect.

where $\boldsymbol{r}_{\mu}+\boldsymbol{u}\left(\boldsymbol{r}_{\mu}, z\right)$ is the real position of the $\mu$-th flux line with $\boldsymbol{u}\left(\boldsymbol{r}_{\mu}, z\right)$ its microscopic displacement field away from the equilibrium position $\boldsymbol{r}_{\mu}$. Variational minimization of the elastic deformation and pinning energies results $\$ 2130$ in an inhomogeneous differential equation; its solution can formally be expressed through a self-consistency condition involving the lattice's elastic Green's function $G_{\alpha \beta}(\boldsymbol{r}, z)$,

$$
\begin{aligned}
& u_{\alpha}\left(\boldsymbol{r}_{\nu}, z_{\nu}\right)= \\
& \quad \int d z d^{2} r G_{\alpha \beta}\left(\boldsymbol{r}_{\nu}-\boldsymbol{r}, z_{\nu}-z\right)\left[-\partial_{u_{\beta}} \varepsilon_{p}(\boldsymbol{r}, z, \boldsymbol{u})\right] .
\end{aligned}
$$

Here, $\alpha$ and $\beta$ index the in-plane components $x$ and $y$, and $\nu$ is a vortex label and we assume summation over double indices. Inserting Eq. (9) into Eq. $(10)$ and defining the pinning force profile $\boldsymbol{f}_{p}(\boldsymbol{r})=-\nabla_{\boldsymbol{r}} e_{p}(\boldsymbol{r})$, we find to dominant order in $\boldsymbol{u}$

$$
u_{\alpha}\left(\boldsymbol{r}_{\nu}, z_{\nu}\right)=\sum_{\mu} G_{\alpha \beta}\left(\boldsymbol{r}_{\nu}-\boldsymbol{r}_{\mu}, z_{\nu}\right) f_{p, \beta}\left[\boldsymbol{r}_{\mu}+\boldsymbol{u}\left(\boldsymbol{r}_{\mu}, 0\right)\right] .
$$

For a pinning potential with a trapping range smaller than the inter-vortex distance $a_{0}$ and pinning at most one vortex, only one term is relevant in the above summation and we arrive at

$$
u_{\alpha}\left(\boldsymbol{r}_{\nu}, z_{\nu}\right)=G_{\alpha \beta}\left(\boldsymbol{r}_{\nu}-\boldsymbol{r}_{p}, z_{\nu}\right) f_{p, \beta}\left[\boldsymbol{r}_{p}+\boldsymbol{u}\left(\boldsymbol{r}_{p}, 0\right)\right],
$$

with $\boldsymbol{r}_{p}=\boldsymbol{r}_{\mu}$ the equilibrium position of the vortex in the vicinity of the pinning site. Evaluating (12) for this vortex at $\boldsymbol{r}_{\nu}=\boldsymbol{r}_{p}$ and $z_{\nu}=0$, we obtain the self-consistency condition

$$
u_{\alpha}\left(\boldsymbol{r}_{p}\right)=G_{\alpha \beta}(\mathbf{0}, 0) f_{p, \beta}\left[\boldsymbol{r}_{p}+\boldsymbol{u}\left(\boldsymbol{r}_{p}\right)\right]
$$

for the displacement $u_{\alpha}\left(\boldsymbol{r}_{p}\right) \equiv u_{\alpha}\left(\boldsymbol{r}_{p}, 0\right)$ of the vortex pinned at the defect site. This expression can be further 
simplified by exploiting the isotropy of the local Green's function, $G_{\alpha \beta}(\mathbf{0}, 0)=\delta_{\alpha \beta} / \bar{C}$, with the effective elasticity $\bar{C}$ defined through

$$
\bar{C}^{-1}=\frac{1}{2} \int_{\mathrm{Bz}} \frac{d^{2} k d k_{z}}{(2 \pi)^{3}} G_{\alpha \alpha}\left(\boldsymbol{k}, k_{z}\right) .
$$

The integration of the reciprocal-space elastic Green's function 21131132

$$
\begin{aligned}
& G_{\alpha \beta}\left(\boldsymbol{k}, k_{z}\right)= \\
& \quad \frac{k_{\alpha} k_{\beta} / k^{2}}{c_{11}\left(\boldsymbol{k}, k_{z}\right) k^{2}+c_{44}\left(\boldsymbol{k}, k_{z}\right) k_{z}^{2}}+\frac{\delta_{\alpha \beta}-k_{\alpha} k_{\beta} / k^{2}}{c_{66} k^{2}+c_{44}\left(\boldsymbol{k}, k_{z}\right) k_{z}^{2}}
\end{aligned}
$$

over the vortex lattice Brillouin zone (BZ) involves the dispersive compression- $\left[c_{11}\left(\boldsymbol{k}, k_{z}\right)\right]$ and tilt- $\left[c_{44}\left(\boldsymbol{k}, k_{z}\right)\right]$ as well as the non-dispersive shear moduli $\left(c_{66}\right)$, see also Ref. 30. Here $k=|\boldsymbol{k}|$ is the norm of the in-plane momentum $\boldsymbol{k}=\left(k_{x}, k_{y}\right)$. Performing the integration in Eq. (14) using Eq. (15) provides us with the expression

$$
\bar{C}=\nu \frac{a_{0}^{2}}{\lambda} \sqrt{c_{66} c_{44}(\mathbf{0}, 0)} .
$$

The determination of the numerical factor $\nu$ requires an accurate evaluation of the linear response of a vortex to a local force. Simple estimates for $\nu$ can be obtained through the approximate evaluation of the integral in Eq. (14) or by calculating the deformation energy of a single flux line embedded in a rigid cage potentia ${ }^{33}$. In the first case, we neglect the compression term in Eq. (15) since $c_{66} \ll c_{11}$. Using $c_{44}\left(\boldsymbol{k}, k_{z}\right)=c_{44}(\mathbf{0}, 0) /\left[1+\lambda^{2}\left(\bar{k}^{2}+k_{z}^{2}\right)\right]$, see Refs. 32] and [30, and assuming $k^{2} \lambda^{2} \gg k_{z}^{2} \lambda^{2}$ and $k^{2} \lambda^{2} \gg 1$, we can extend the integral over $k_{z}$ to infinity and limit the planar integral to the circularized Brillouin zone $k^{2}<4 \pi / a_{0}^{2}$. Using these approximations, we arrive at a numerical $\nu=4$. The alternative estimate is based on a flux line with elasticity $\varepsilon_{l}=\varepsilon_{0}$ trapped within a cage potential $V_{\text {cage }}=\pi \varepsilon_{0}\left(u / a_{0}\right)^{2}$ set up by the neighboring vortices 33 , where $\varepsilon_{0}=\left(\Phi_{0} / 4 \pi \lambda\right)^{2}$ denotes the vortex line energy. Minimizing the total en$\operatorname{ergy~} \int d z\left[\varepsilon_{l} u^{2} / 2+V_{\text {cage }}(u)\right] \equiv \bar{C} u^{2} / 2$, we obtain $\bar{C}=$ $4 \sqrt{2 \pi} \varepsilon_{0} / a_{0}$, which corresponds to a factor $\nu=4 \sqrt{2}$ when recast into the form 16 .

Making use of the effective elasticity $\bar{C}$, Eq. (13) can be written in the form

$$
\bar{C} \boldsymbol{u}(\boldsymbol{r})=\boldsymbol{f}_{p}[\boldsymbol{r}+\boldsymbol{u}(\boldsymbol{r})],
$$

where we have dropped the subscript in the vortex-pin distance, $\boldsymbol{r}_{p} \rightarrow \boldsymbol{r}$. It is the appearance of multiple solutions of this non-linear self-consistency equation which is at the origin of the strong pinning phenomenon. Inserting the solution $\boldsymbol{u}(\boldsymbol{r})$ of Eq. (17) equation back into Eq. (12), the displacement field $u_{\alpha}\left(\boldsymbol{r}_{\nu}, z_{\nu}\right)$ of all vortices can be determined.

The self-consistency equation (17) is easily derived as the minimizer of the total free energy including contributions from elasticity and pinning 34 ,

$$
e_{\text {pin }}(\boldsymbol{r})=\frac{1}{2} \bar{C} u^{2}+e_{p}(\boldsymbol{r}+\boldsymbol{u}) .
$$

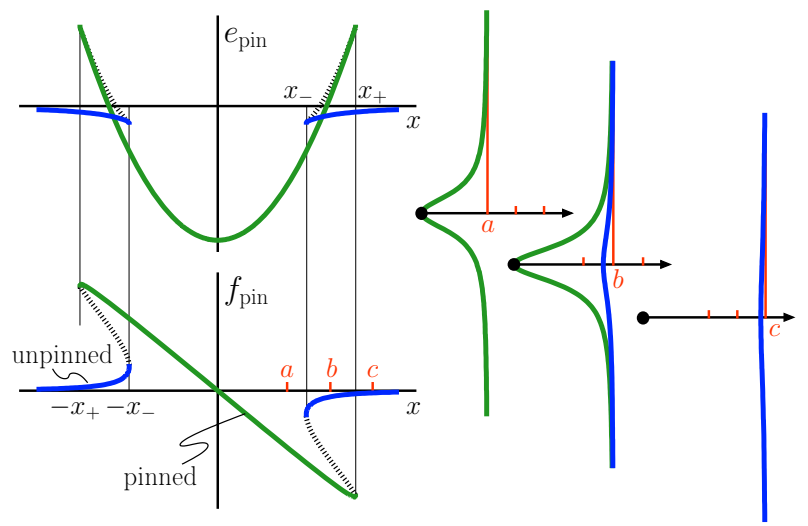

FIG. 2. Left: Energy- and force profiles at strong pinning $\kappa>1$ with multi-valued solutions within the intervals $\left[-x_{+},-x_{-}\right]$and $\left[x_{-}, x_{+}\right]$. Stable pinned (green) and unpinned (blue) branches are connected by unstable solutions (dashed). Right: Vortex shapes associated with pinned ( $a, b$ in green) and unpinned ( $b, c$ in blue) branches for different positions away from the pin.

Indeed, minimizing Eq. (18) with respect to the displacement $\boldsymbol{u}$ leads to Eq. (17). On the other hand, the derivative with respect to $\boldsymbol{r}$ produces the effective force profile

$$
\boldsymbol{f}_{\text {pin }}(\boldsymbol{r}) \equiv-\nabla_{\boldsymbol{r}} e_{\text {pin }}(\boldsymbol{r})
$$

associated with the total energy (18): evaluating the total derivative $\nabla_{\boldsymbol{r}} e_{\mathrm{pin}}(\boldsymbol{r})$, we can express the gradient $-\nabla_{\boldsymbol{r}} e_{p}(\boldsymbol{r})$ through the bare pinning force, $-\nabla_{\boldsymbol{r}} e_{p}(\boldsymbol{r})=$ $\boldsymbol{f}_{p}(\boldsymbol{r})$, and making use of Eq. (17), we find that

$$
\boldsymbol{f}_{\mathrm{pin}}(\boldsymbol{r})=\boldsymbol{f}_{p}[\boldsymbol{r}+\boldsymbol{u}(\boldsymbol{r})]=\bar{C} \boldsymbol{u}(\boldsymbol{r}) .
$$

Hence, a multi-valued solution of Eq. (17) at a given $\boldsymbol{r}$ entails multi-valued solutions for the energy profile $e_{\text {pin }}(\boldsymbol{r})$ as well as the force profile $\boldsymbol{f}_{\text {pin }}(\boldsymbol{r})$.

For the geometry introduced in Sec. III and discussed below, we simplify the formalism further by assuming that all vortex trajectories with impact parameter $2|y|<$ $t_{\perp}$ experience maximal pinning, i.e., that of a vortex hitting the defect head-on with $y=0$. For a small pinning center, the transverse length $t_{\perp}$ is of the order of the vortex core size $\xi$ (the coherence length), while $t_{\perp}$ is determined by the pin size for a large defect. With this simplification, the problem reduces to one effective dimension with Eqs. (17) and (19) taking the form

$$
\begin{aligned}
\bar{C} u(x) & =f_{p}[x+u(x)]=f_{\text {pin }}(x), \quad \text { with } \\
f_{\text {pin }}(x) & \equiv-d e_{\text {pin }}(x) / d x .
\end{aligned}
$$

The self-consistency equation 21) can be easily tested for multi-valued solutions; these appear when the derivative $d u / d x \equiv u^{\prime}(x)$ turns infinite. Taking the total derivative of Eq. (21) with respect to $x$, we find that

$$
u^{\prime}(x)=\frac{1}{\bar{C} / f_{p}^{\prime}[x+u(x)]-1}
$$


diverges with increasing pinning force for the first time when the maximal force derivative $f_{p}^{\prime}$ matches the elasticity $\bar{C}$. This onset of strong pinning then is defined by the Labusch criterion $\kappa=1$, where $\kappa \equiv \max _{x}\left[f_{p}^{\prime}(x) / \bar{C}\right]$ denotes the Labusch parameter. At small values of $\kappa<1$, i.e., when $f_{p}^{\prime}(x)<\bar{C}$ for any $x$, the force profile $f_{\text {pin }}(x)$ is single valued and $u^{\prime}(x)$ always has the same sign as $f_{p}^{\prime}[x+u(x)]$. Within the strong pinning framework, this weak pinning regime $\kappa<1$ is associated with a vanishing average pinning force $F_{\text {pin }}=0$ and hence $j_{c}=0$. At the same time, the absence of force jumps is associated with a divergent Campbell length $\lambda_{\mathrm{C}}=\infty$, see below. These results are modified if collective phenomena are included in the model, a topic that goes beyond the present work.

For $\kappa>1$, the pinning force profile $f_{\text {pin }}(x)$ turns multivalued with inflection points at $\pm x_{-}$and $\pm x_{+}(0<$ $\left.x_{-}<x_{+}\right)$where Eq. (23) diverges, see Fig. 2 . Between the two inflection points $x_{ \pm}$, two stable and one unstable branch exist, the latter being characterized by $u^{\prime}(x) / f_{p}^{\prime}[x+u(x)]<0$. The two stable branches are smoothly connected to a vortex trapped by the pin and a vortex detached from the pin, respectively. Hence, we shall use the terminology 'pinned' and 'unpinned' branches for these two solutions. The pinning region $|\boldsymbol{r}|<x_{-}$, where only the pinned branch exists, defines the transverse trapping length $t_{\perp}=2 x_{-}$. For strong pinning, the pinscape produces a finite macroscopic pinning force density by asymmetrically populating the different branches of $f_{\text {pin }}(x)$.

Hence, the Labusch criterion ${ }^{27} \kappa=f_{p}^{\prime}\left(x_{m}\right) / \bar{C}=1$, with $\max _{x}\left[f_{p}^{\prime}(x)\right]=f_{p}^{\prime}\left(x_{m}\right)$ realized at $x_{m}$, serves as a quantitative separation between the regimes of weak $(\kappa<1)$ and strong $(\kappa>1)$ pinning. As pinning vanishes alltogether at $\kappa<1$, one often uses the distinction between weak, intermediate, and strong 'strong-pinning' regimes with different $j_{c}$-scalings, $j_{c} \propto(\kappa-1)^{2}$ and $j_{c} \propto \kappa^{2}$, at the two extremes. With the formalism of strong pinning at hand, we are now ready to discuss the physical implications of vortex pinning by a low density of pinning centers.

\section{B. Critical current}

For randomly (and homogeneously) distributed pinning sites with a small density $n_{p}$ (see below for a quantitative criterion), the macroscopic pinning force density $F_{\text {pin }}$ results from proper averaging of the microscopic forces 22,

$$
F_{\mathrm{pin}}=n_{p}\left\langle f_{\mathrm{pin}}\right\rangle=\left.n_{p} \frac{t_{\perp}}{a_{0}} \int \frac{d x}{a_{0}} f_{\mathrm{pin}}(x)\right|_{\mathrm{o}}
$$

with $\left.f_{\text {pin }}(x)\right|_{\mathrm{o}} \equiv f_{p}\left[x+u^{\mathrm{o}}(x)\right]$ referring to the occupied branches $u^{\mathrm{o}}$ in the effective force profile $f_{\text {pin }}(x)$. In the zero-field-cooled (critical) state, the pinning landscape acts with the critical force density $F_{\text {pin }}=-F_{c}$ against the Lorentz force density $j B_{0} / c$, thus defining the critical current density $j_{c}=c F_{c} / B_{0}$. This maximal pinning force density is achieved when the pinned branch $u^{\mathrm{p}}(x)$ is occupied in a maximally asymmetric way between $-x_{-}$ to $x_{+}$, see Fig. 3. Combining Eqs. (22) and (24), we arrive at a microscopic expression for the critical current $j_{c}=-\left(c / B_{0}\right) F_{c}$,

$$
j_{c}=\frac{c n_{p} t_{\perp}}{\Phi_{0}} \Delta e_{\mathrm{pin}}
$$

where $\Delta e_{\text {pin }}$ denotes the sum of the jumps in $e_{\text {pin }}(x)$ between pinned and unpinned branches at the positions $-x_{-}$and $x_{+}$. More precisely,

$$
\Delta e_{\mathrm{pin}}=\left.\left(e_{\mathrm{pin}}^{\mathrm{up}}-e_{\mathrm{pin}}^{\mathrm{p}}\right)\right|_{-x_{-}}+\left.\left(e_{\mathrm{pin}}^{\mathrm{p}}-e_{\mathrm{pin}}^{\mathrm{up}}\right)\right|_{x_{+}},
$$

where the superscripts 'up' and 'p' denote unpinned and pinned branches. While Eq. 25) provides a quantitative expression for the critical current density within strong pinning theory, we may use $t_{\perp} \sim \xi$ and $\Delta e_{\text {pin }} \sim f_{p} x_{+} \sim$ $f_{p}^{2} / \bar{C}$ (with $f_{p}$ the typical strength of the bare pinning force and $x_{+} \sim \kappa \xi$ ) to arrive at a qualitative estimate for the critical current, $j_{c} \sim c n_{p} \xi f_{p}^{2} / \Phi_{0} \bar{C}$. Together with the scaling $\bar{C} \propto\left(\Phi_{0} / 4 \pi \lambda\right)^{2} / a_{0}=\varepsilon_{0} / a_{0}$ of the effective elasticity at low fields, we arrive at

$$
j_{c} \sim j_{\mathrm{dp}}\left(n_{p} a_{0} \xi^{2}\right)\left(\kappa \xi / a_{0}\right)^{2},
$$

with the depairing current $j_{\mathrm{dp}}=c \Phi_{0} /\left(12 \sqrt{3} \pi^{2} \lambda^{2} \xi\right)$ and the small parameter $n_{p} a_{0} \xi^{2} \ll 1$ defining the regime of 3D strong pinning, see Ref. 22. The field scaling $j_{c} \propto$ $1 / \sqrt{B_{0}}$ is in agreement with the results obtained in the early work on strong pinning by Ovchinnikov and Ivlev $\sqrt{35}$.

\section{Campbell Length}

The Campbell penetration depth $\lambda_{\mathrm{C}}$ is another measureable quantity characterizing the pinning landscape. In a microscopic derivation of $\lambda_{\mathrm{C}}$, we have to find the dynamical change in pinning force $\delta F_{\text {pin }}[U(X, t)]$. The latter is determined by the change in branch occupation due to the macroscopic displacement $U$ of the vortex lattice. As shown below, the macroscopic Campbell length relates to $\Delta f_{\text {pin }}$, the sum of the jumps in $f_{\text {pin }}(x)$ between occupied and unoccupied branches,

$$
\frac{1}{\lambda_{\mathrm{C}}^{2}}=\frac{4 \pi n_{p} t_{\perp}}{B_{0} \Phi_{0}} \Delta f_{\mathrm{pin}}
$$

and hence probes another quantity than $j_{c}$, Eq. (25), Beloow, we derive this result for two initial states of particular importance, the zero-field-cooled (or Bean critical) state and the field-cooled state.

\section{Bean critical state}

The Bean critical state, as described in Sec. IIIB is characterized by the maximal or critical pinning force 
$F_{\text {pin }}=-F_{c}$ with an asymmetric branch occupation. Hence, depending on the sign of $U$, the branch occupation will be affected differently. Specifically, for a macroscopic shift of all vortices in the direction of the Lorentz force, i.e., $x \rightarrow x+U$ with $U>0$, most vortices adiabatically follow their branch, $u(x) \rightarrow u(x+U)$. The few vortices on the unpinned (pinned) branch at distances less than $U$ away from the branch edge at $-x_{-}\left(x_{+}\right)$will be pushed beyond that boundary and irreversibly jump to the pinned (unpinned) solution, see inset of Fig. 3. Hence, a displacement $U>0$ leads to (i) a net penetration of vortices into the sample while (ii) leaving the branch occupation unchanged, i.e.,

$$
\delta F_{\text {pin }}(U>0)=0 .
$$

On the other hand, for a displacement $x \rightarrow x+U$ to the left with $U<0$, i.e., against the critical slope, all vortices adiabatically follow their branches. The occupation of the pinned branch then is shifted to lie between $-x_{-}+U$ and $x_{+}+U$, see Fig. 3. Similarly, the unpinned branch is occupied until $-x_{-}+U$ and onwards from $x_{+}+U$. The change in pinning force $\delta F_{\text {pin }}(U)$ is obtained from the difference of the pinning force (24) evaluated for the critical state shifted by $U$, leading to

$$
\begin{gathered}
\delta F_{\text {pin }}(U<0)=\frac{n_{p} t_{\perp}}{a_{0}^{2}}\left[\int_{-x_{-}+U}^{-x_{-}} d x\left[f_{\text {pin }}^{\mathrm{p}}(x)-f_{\text {pin }}^{\mathrm{up}}(x)\right]\right. \\
\left.\quad+\int_{x_{+}+U}^{x_{+}} d x\left[f_{\text {pin }}^{\mathrm{up}}(x)-f_{\text {pin }}^{\mathrm{p}}(x)\right]\right] \\
\approx-\frac{n_{p} t_{\perp}}{a_{0}^{2}}\left[\left.\left(f_{\mathrm{pin}}^{\mathrm{p}}-f_{\text {pin }}^{\mathrm{up}}\right)\right|_{-x_{-}}+\left.\left(f_{\text {pin }}^{\mathrm{up}}-f_{\mathrm{pin}}^{\mathrm{p}}\right)\right|_{x_{+}}\right] U \\
=-\frac{n_{p} t_{\perp}}{a_{0}^{2}} \Delta f_{\text {pin }} U .
\end{gathered}
$$

This drop in the critical force always appears when vortices start moving to the left and is associated with a reduction of $U(X, t)$ with increasing time. In order to follow dynamically the appearance and disappearance of this term in the equation of motion, we introduce the max-field $U_{0}(X, t)=\max _{t^{\prime}<t} U(X, t)$. Whenever $U(X, t)$ changes the direction of motion from right to left, $U$ starts deviating from $U_{0}$. The argument $U$ in Eq. (31) should then be replaced by $U-U_{0}$. In the end, the piecewise change of the pinning force entering the macroscopic equation of motion (5) reduces to the simple expression

$$
\delta F_{\text {pin }}(U)=-\alpha_{\text {sp }}\left(U-U_{0}\right),
$$

and satisfies both Eqs. (29) and (31). In the above expression, the underlying pinning potential enters solely through the coefficient $\alpha_{\mathrm{sp}}=\left(n_{p} t_{\perp} / a_{0}^{2}\right) \Delta f_{\text {pin }}$. This coefficient can be understood as the mean curvature of the pinning energy,

$$
\alpha_{\mathrm{sp}}=n_{p}\left\langle e_{\mathrm{pin}}^{\prime \prime}\right\rangle=-\left.\frac{n_{p} t_{\perp}}{a_{0}} \int \frac{d x}{a_{0}} f_{\mathrm{pin}}^{\prime}(x)\right|_{\mathrm{o}},
$$

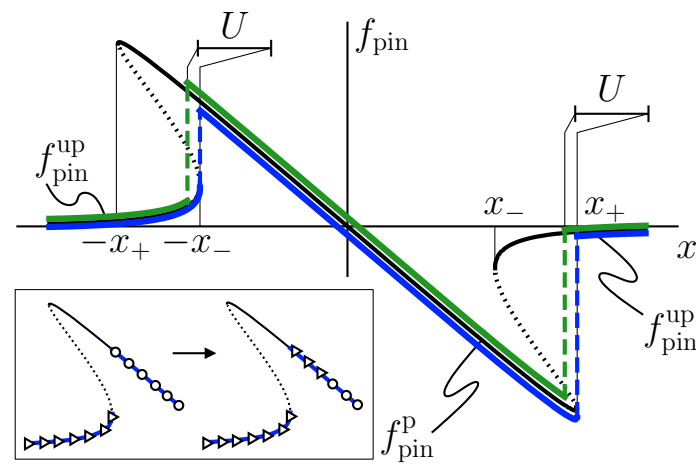

FIG. 3. Occupation of the pinned $\left(f_{\text {pin }}^{\mathrm{p}}\right)$ and unpinned $\left(f_{\text {pin }}^{\text {up }}\right)$ branches in the critical state (blue). A macroscopic displacement of all vortices to the left, $U<0$, results in a new branch occupation (green), with vortices populating the pinned (unpinned) branch below $-x_{-}\left(x_{+}\right)$while the corresponding states on the unpinned (pinned) branch get depleted; this change in occupation leads to a decrease of the macroscopic pinning force density. On the other hand, for a uniform shift of all vortices to the right with $U>0$, vortices within a distance $U$ to the left of the branch edge irreversibly jump to the other stable solution (see inset). This process does not lead to a net change in branch occupation and the pinning force remains at its critical value.

similar to the mean force in Eq. 24) defining the critical current density $j_{c}$.

The asymmetric response of the vortex system to an increasing versus decreasing field is associated with a peculiar transient initialization towards a periodic vortex motion, where on every $a c$ cycle vortices are pumped (and diffusively penetrate) into the sample, asymptotically changing the $d c$ field from $B_{0}$ to $B_{0}+h_{a c}$ after many cycles. A detailed discussion of this process is presented in Ref. 26], where it is shown that the number of cycles needed to shift the critical state from $B_{0}$ to $B_{0}+h_{a c}$ within the depth of the Bean profile $L=c B_{0} / 4 \pi j_{c}$ is about $N=\left(\pi L / 2 \ell_{D}\right)^{2}$, with $\ell_{D}=\left(B_{0}^{2} / 2 \omega \eta\right)^{1 / 2}$ the diffusion length per cycle period $2 \pi / \omega$. After this rectification process, vortices move reversibly in their wells as $U$ always remains below $U_{0}$. The latter reaches the asymptotic form $U_{0}(X)=\left(h_{a c} / B_{0}\right)(L-X)$. For a sample of finite thickness $d<2 L$ along $X, d / 2$ replaces $L$ is the expressions for $N$ and $U_{0}$. The reversible dynamics of vortices after the initialization can be solved by substituting the variable $\delta U(X, t) \equiv U(X, t)-U_{0}(X)$ into Eq. (5) and one finds

$$
\delta U(X, t)=-\lambda_{\mathrm{C}}\left(h_{a c} / B_{0}\right) e^{-X / \lambda_{\mathrm{C}}}\left[1-e^{-i \omega t}\right] .
$$

The $a c$ response of the vortex lattice in the critical state

$$
\delta B(X, t)=h_{a c} e^{-X / \lambda_{\mathrm{C}}} e^{-i \omega t}+h_{a c}\left[1-e^{-X / \lambda_{\mathrm{C}}}\right]
$$

is regular and involves the Campbell length $\lambda_{\mathrm{C}}$ given by Eq. (28). The asymptotic solution consists of an oscillatory response within a surface layer $\sim \lambda_{\mathrm{C}}$ and a rectified $d c$ part that has penetrated deep into the bulk. This behavior is very similar to the critical $a c$ response discussed 
by Bean ${ }^{18}$ (Bean penetration), where large amplitude oscillations $h_{a c} \gg j_{c} \lambda_{\mathrm{C}} / c$ generate a nonlinear response. An extended comparison between these two scenarios is given in Ref. [26].

\section{Field-cooled state}

The field-cooled state is characterized by vanishing net currents and net pinning forces. In the strong pinning regime, the vanishing pinning force translates into a symmetric occupation of the branches, with jumps between the pinned and unpinned branch located at $\pm x_{\mathrm{jp}} \in\left[x_{-}, x_{+}\right]$. If this position is away from the branch edges $x_{ \pm}$, the oscillation is always reversible. If $x_{\mathrm{jp}}$ coincides with one of the branch edges $x_{ \pm}$, a one-cycle initialization process reshuffles few vortices near the branch edges, after which the oscillation is reversible and the result in Eq. 28 involves the jumps at $\pm x_{ \pm}$. Hence, no complex initialization process needs to be studied for the field-cooled situation and the change in the pinning force is always given by the expression

$$
\delta F_{\text {pin }}(U)=-\frac{n_{p} t_{\perp}}{a_{0}^{2}} \Delta f_{\text {pin }} U,
$$

with $\Delta f_{\text {pin }}$ now involving two identical jumps at $\pm x_{\mathrm{jp}}$.

In order to quantify the Campbell length in the fieldcooled state, the central remaining task is to determine the precise position of the jump $x_{\mathrm{jp}}$ within the interval $\left[x_{-}, x_{+}\right]$and find the corresponding jump $\Delta f_{\text {pin }}$ upon changing the temperature $T$. For insulating or metallic inclusions, where pinning smoothly increases when crossing $H_{c 2}(T)$ (see below), we can follow the branch occupation as a function of $T$ as the system evolves from weak $(\kappa<1)$ to strong $(\kappa>1)$ pinning through the Labusch point $\kappa\left(T_{\mathrm{L}}\right)=f_{p}^{\prime}\left(x_{m}\right) /\left.\bar{C}\right|_{T_{\mathrm{L}}}=1$ defining the Labusch temperature $T_{\mathrm{L}}$ (here, $x_{m}$ denotes the point of maximal slope $\left.f_{p}^{\prime}\right)$. Above the Labusch temperature, $T>T_{\mathrm{L}}$, the force profile is single valued, the critical current vanishes [see Eq. [25)], and the Campbell length is formally infinite [see Eq. (28)] due to the absence of jumps. These singular results are regularized once collective pinning effects are considered. Upon lowering the temperature, the system reaches the Labusch point $\kappa\left(T_{\mathrm{L}}\right)=1$, where the pinning force $f_{\text {pin }}$ develops a vertical slope at $x_{0 \mathrm{~L}}$, $f_{p}^{\prime}\left[x_{0 \mathrm{~L}}+u\left(x_{0 \mathrm{~L}}\right)\right]=\bar{C}$, see Eq. [23). The combination with Eq. 21) and the Labusch criterion $f_{p}^{\prime}\left(x_{m}\right)=\bar{C}$ provides us with the relation $x_{0 \mathrm{~L}}=x_{m}-f_{p}\left(x_{m}\right) /\left.\bar{C}\right|_{T_{\mathrm{L}}}$. Lowering the temperature $T$ further below $T_{\mathrm{L}}$, the pinning force $f_{\text {pin }}$ turns multi-valued within the intervals $\pm\left[x_{-}, x_{+}\right]$, see Fig. 2 .

Depending on the temperature dependence of the elastic and pinning forces, we have identified three possible scenarios defining the (symmetric) jump positions $\pm x_{\mathrm{jp}}$ in the branch occupation, see Fig. 4 In the first case (a), the branch edges $x_{ \pm}$move away from $x_{0 \mathrm{~L}}$ in opposite directions, $x_{-}<x_{0 \mathrm{~L}}<x_{+}$. The second case (b), describes the situation where both boundaries $x_{ \pm}$become

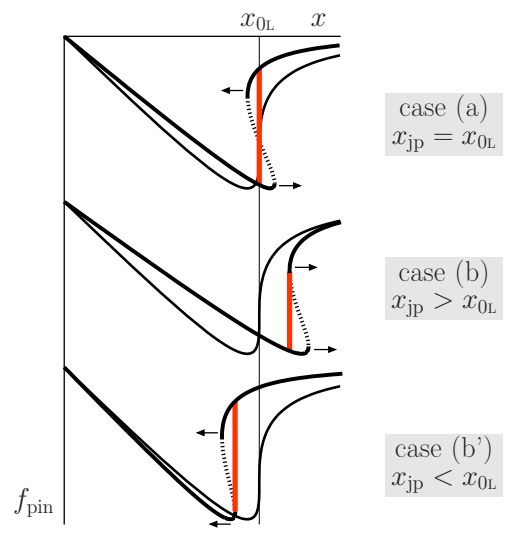

FIG. 4. Upon lowering the temperature below the Labusch temperature, $T<T_{\mathrm{L}}$, the force profile turns multi-valued with the bistable region centered about $x_{0 \mathrm{~L}}$ [case (a)], to its right [case (b)], or to its left [case (b')]. Specific microscopic pinning mechanisms (see text) entail one of these three cases, each associated with its force jump at $x_{\mathrm{jp}}$. These force jumps $\Delta f_{\text {pin }}$ (red lines) are probed by a measurement of the Campbell length $\lambda_{\mathrm{C}}$.

larger than $x_{0 \mathrm{~L}}$ upon cooling, $x_{0 \mathrm{~L}}<x_{-}<x_{+}$, while they become smaller in the third case ( $\left.\mathrm{b}^{\prime}\right), x_{-}<x_{+}<x_{0 \mathrm{~L}}$.

In the simplest case $(\mathrm{a})$, vortices at $x<x_{0 \mathrm{~L}}\left(x>x_{0 \mathrm{~L}}\right)$ follow adiabatically the evolution of the pinned (unpinned) branch and the occupation jumps at $x_{\mathrm{jp}}=x_{0 \mathrm{~L}}$, such that $\Delta f_{\text {pin }}=\left.2 \Delta f_{\text {pin }}\right|_{x_{0 \mathrm{~L}}}$ enters the expression (28) for the Campbell length, with $\left.\Delta f_{\text {pin }}\right|_{x_{0 \mathrm{~L}}}$ denoting the jump in $f_{\text {pin }}$ at $x_{0 \mathrm{~L}}$. In case (b), the unpinned branch, initially existing for $x>x_{0 \mathrm{~L}}$, becomes unstable in the interval $\left[x_{0 \mathrm{~L}}, x_{-}\right]$. As a result, vortices with $x_{0 \mathrm{~L}}<x<x_{-}$ now occupy the pinned branch and the force jump appears at $x_{\mathrm{jp}}=x_{-}$, with $\Delta f_{\text {pin }}=\left.2 \Delta f_{\text {pin }}\right|_{x_{-}}$entering $\lambda_{\mathrm{C}}$. Similarly, in case (b'), the vortices populate the unpinned branch in the interval $\left[x_{+}, x_{0 \mathrm{~L}}\right]$ where the pinned solution has stopped existing. The Campbell length then involves the jumps at $\pm x_{+}$.

The repopulation of vortices from the unpinned to the pinned branch in case (b) [or vice-versa in case ( $\left.\mathrm{b}^{\prime}\right)$ ] leads to a hysteretic response if the system is reheated after the cooling process. Consider a system in case (b) cooled to the minimal temperature $T_{\min }$ and subsequently reheated. Upon cooling, vortices on the unpinned branch become locally unstable at $x_{-}(T)$ and the jump in occupation follows $x_{-}(T)$; the Campbell length Eq. (28) involves $\Delta f_{\text {pin }}=\left.2 \Delta f_{\text {pin }}\right|_{x_{-}(T)}$, see the discussion above and Fig. 5. Upon reversing the temperature sweep at $T_{\min }$, the jump is locked to $x_{-}\left(T_{\min }\right)$ as all vortices remain stable within their branches; the Campbell length now involves the jumps $\Delta f_{\text {pin }}=\left.2 \Delta f_{\text {pin }}\right|_{x_{-}\left(T_{\min }\right)}$. With the temperature increasing further, vortices on the pinned branch become locally unstable at $x_{+}(T) \leq$ $x_{-}\left(T_{\min }\right)$ and the jump in occupation follows $x_{+}(T)$; the Campbell length then involves the jumps $\Delta f_{\text {pin }}=$ $\left.2 \Delta f_{\text {pin }}\right|_{x_{+}(T)}$. The difference in the force jumps then naturally leads to a hysteretic behavior of the Campbell 
length $\lambda_{\mathrm{C}}$.

In order to provide a quantitative insight into the evolution of $x_{ \pm}$away from (but close to) $x_{0 \mathrm{~L}}$, we expand the bare pinning force around $x_{m}$,

$$
f_{p}(x) \approx f_{p}\left(x_{m}\right)+f_{p}^{\prime}\left(x_{m}\right)\left(x-x_{m}\right)-\frac{\gamma}{3}\left(x-x_{m}\right)^{3},
$$

with $f_{p}^{\prime \prime}\left(x_{m}\right)=0$ and $\gamma \equiv-f_{p}^{\prime \prime \prime}\left(x_{m}\right) / 2>0$. These two conditions originate from the definition of $x_{m}$ as the location maximizing $f_{p}^{\prime}(x)$. Using the above expression in combination with Eqs. 21) and (22), allows us to find the inflection points $x_{ \pm}$in $u(x)$ [or $f_{\text {pin }}(x)$ ] characterized through a vanishing denominator on the right-hand side of Eq. (23). A straightforward calculation provides the result

$$
x_{ \pm}=x_{0} \pm \frac{2}{3} \sqrt{\frac{\bar{C}}{\gamma}}(\kappa-1)^{3 / 2}
$$

where $x_{0}(T)=x_{m}-f_{p}\left(x_{m}\right) /\left.\bar{C}\right|_{T}$ is a generalization of $x_{0 \mathrm{~L}}=x_{0}\left(T_{\mathrm{L}}\right)$. Case (a) is realized when $x_{0}(T)=x_{0 \mathrm{~L}}$ to order $(\kappa-1)^{2}$, i.e., the relevant jump is at $x_{0 \mathrm{~L}}$. Solving the self-consistency equation 21$)$ for $u_{ \pm}\left(x_{0 \mathrm{~L}}\right)$ and using

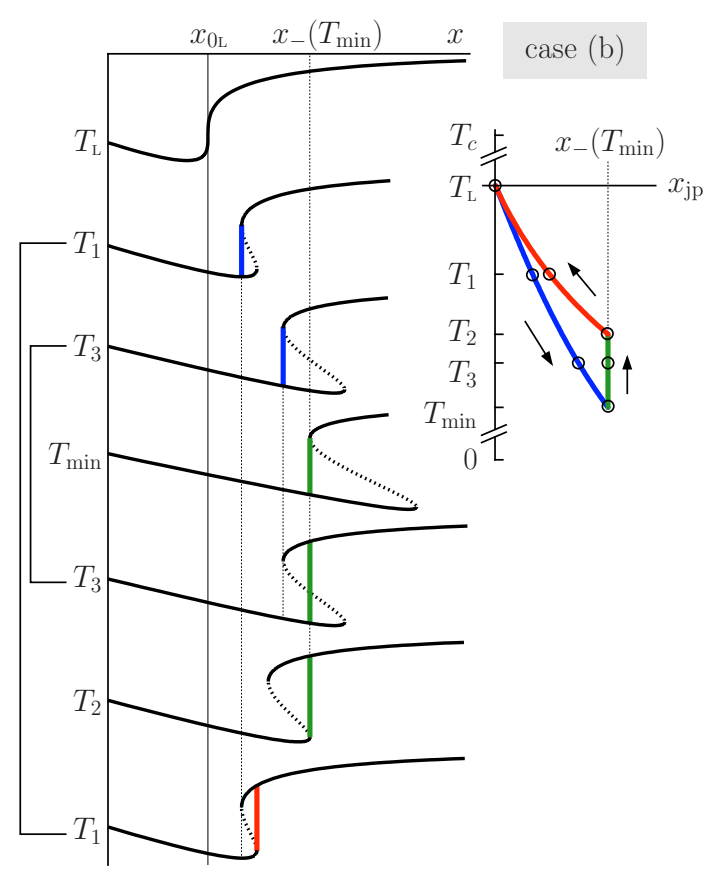

FIG. 5. Illustration of the evolution of the force profile at different temperatures $T_{\mathrm{L}}>T_{1}>T_{2}>T_{3}>T_{\min }$ [case (b) in Fig. 4], and the associated hysteresis in the force jump position $x_{\mathrm{jp}}$ (inset) upon cooling from $T_{\mathrm{L}}$ down to $T_{\min }$ and subsequent heating. Upon cooling, the force jump probed by the Campbell length is positioned at the branch edge $x_{\mathrm{jp}}=x_{-}$ (blue). During reheating, the jump's position first remains fixed at $x_{\mathrm{jp}}=x_{-}\left(T_{\min }\right)$ (green). At the temperature $T_{2}$, the edge at $x_{+}$coincides with the position $x_{-}$previously reached at $T_{\min }$. When the temperature increases above $T_{2}$ the force jump follows the other branch edge at $x_{+}$(red). the relation $\left.\Delta u\right|_{x_{0 \mathrm{~L}}} \equiv\left[u_{+}-u_{-}\right]_{x_{0 \mathrm{~L}}}=\left.\Delta f_{\mathrm{pin}}\right|_{x_{0 \mathrm{~L}}} / \bar{C}$, we find the force jump

$$
\left.\Delta f_{\text {pin }}\right|_{x_{0 \mathrm{~L}}}=2 \sqrt{3} \bar{C} \sqrt{\bar{C} / \gamma}(\kappa-1)^{1 / 2} .
$$

However, case (a) is a special situation since the term linear in $(\kappa-1)$ in the expansion of $x_{0}$, see Eq. (38), has to vanish. For the generic cases (b) and (b') the relevant jumps are at $x_{-}$and $x_{+}$, respectively. Solving the cubic self-consistency equation Eq. (21) for $u\left(x_{+}\right)$, we find one doubly degenerate solution $u_{+}$for the deformation at the edge of the pinned branch and a non-degenerate solution $u_{-}$within the unpinned branch. The force jump at $x_{+}$ then amounts to $\bar{C}\left|u_{+}-u_{-}\right|$. A similar analysis can be carried out for $u\left(x_{-}\right)$; to this order in the expansion (37), both jumps turn out identical and take the form

$$
\left.\Delta f_{\mathrm{pin}}\right|_{x_{ \pm}}=3 \bar{C} \sqrt{\bar{C} / \gamma}(\kappa-1)^{1 / 2} .
$$

Within the same approximation, the Campbell length in the Bean critical state involves one jump at $-x_{-}$and one at $x_{+}$; since both jumps are equal, the result for the zero-field-cooled state coincides with that for the fieldcooled states of cases (b) and (b'). On the other hand, the non-generic case (a) features a smaller Campbell length since the associated jump centered between $x_{-}$and $x_{+}$ is larger by a factor $2 / \sqrt{3} \approx 1.155$.

Away from the Labusch point, the degeneracy of the cases (b) and ( $\left.b^{\prime}\right)$ is removed and all force jumps are different, with $\left.\lambda_{\mathrm{C}}\right|_{\mathrm{FC}\left(\mathrm{b}^{\prime}\right)}<\left.\lambda_{\mathrm{C}}\right|_{\mathrm{ZFC}}<\left.\lambda_{\mathrm{C}}\right|_{\mathrm{FC}(\mathrm{b})}$. Simple expressions can be provided in the limit $\kappa \gg 1$, where the force jump at $x_{\mathrm{jp}}$ assumes the approximate value $\left.\Delta f_{\mathrm{pin}}\right|_{x_{\mathrm{p}}} \simeq \bar{C} x_{\mathrm{jp}}$ (note that the pinned branch is well described by $f_{\text {pin }}=-\bar{C} x$ away from the Labusch point). These jumps appear at $x_{+}=\kappa \xi$ for case (b') and $x_{0 \mathrm{~L}} \simeq \xi$ for case (a). The jump at $x_{-}(\kappa)$ for case (b) depends on the tail of the force profile $f_{p}(x)$ far from the pin. For a power-law decay $f_{p}(x) \propto-(\xi / x)^{n}$, we find $x_{-}(\kappa) \simeq \xi \kappa^{1 /(n+1)}$, while for an exponential tail $f_{p}(x) \propto-\exp (-x / \xi)$, we have $x_{-}(\kappa) \simeq \xi \ln (\kappa)$. We then find

$$
\frac{\left.\lambda_{\mathrm{C}}^{2}\right|_{\mathrm{FC}\left(\mathrm{b}^{\prime}\right)}}{\left.\lambda_{\mathrm{C}}^{2}\right|_{\mathrm{ZFC}}} \simeq \frac{1}{2}, \quad \frac{\left.\lambda_{\mathrm{C}}^{2}\right|_{\mathrm{FC}(\mathrm{b})}}{\left.\lambda_{\mathrm{C}}^{2}\right|_{\mathrm{ZFC}}} \simeq \frac{\kappa \xi}{2 x_{-}(\kappa)}, \quad \frac{\left.\lambda_{\mathrm{C}}^{2}\right|_{\mathrm{FC}(\mathrm{a})}}{\left.\lambda_{\mathrm{C}}^{2}\right|_{\mathrm{ZFC}}} \simeq \frac{\kappa}{2},
$$

with

$$
\frac{1}{\left.\lambda_{\mathrm{C}}^{2}\right|_{\mathrm{ZFC}}} \simeq \frac{4 \pi n_{p} t_{\perp}}{B_{0} \Phi_{0}} \bar{C} \kappa \xi
$$

If one neglects the weak dependence of $t_{\perp}=2 x_{-}$on $\kappa$, the two last relations in (41) tell that the information on the pinning force $f_{p}$ disappears from the Campbell length in the field-cooled cases (a) and (b). More precisely, following the discussion above, the residual weak dependence of $x_{-}$on $\kappa$ provides information on the decay of $f_{p}(x)$ at large $x>\xi$.

Applying the same estimates as in Sec. IIIB to the zero-field-cooled Campbell length in Eq. (42), we arrive 


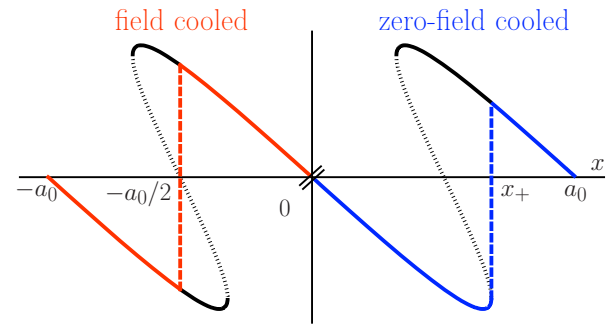

FIG. 6. Branch occupation at high fields when the vortex system is prepared in the field-cooled (left) and zero-fieldcooled (right) states. At high fields, the unpinned branch has disappeared and the Campbell length involves a single jump in force $\Delta f_{\text {pin }}=\left.\Delta f_{\text {pin }}\right|_{x_{\mathrm{jp}}}$ at $x_{\mathrm{jp}}=-a_{0} / 2\left(x_{\mathrm{jp}}=x_{+}\right)$ indicated with a blue (red) dashed line for the (zero)-fieldcooled system. Coordinates are equivalent modulo $a_{0}$.

at the qualitative scaling

$$
\left.\lambda_{\mathrm{C}}^{2}\right|_{\mathrm{ZFC}} \sim \frac{\lambda^{2}}{n_{p} a_{0} \xi^{2} \kappa}>\lambda^{2} .
$$

Similar to the critical current, see Sec. IIIB, the Campbell length involves the small parameter $n_{p} a_{0} \xi^{2} \ll 1$ characteristic of the 3D strong pinning limit 22 .

\section{High fields}

In the discussion above, we have analyzed the interaction of pinning centers with a single flux line. In high fields, where the vortex separation $a_{0}$ is comparable to the maximal pinning length $x_{+} \sim \kappa \xi$, this picture needs to be modified as the periodicity of the pinning potential has to be properly accounted for. In the vicinity of $H_{c 2}$, the pinning potenial is dominanted by the lowest harmonic, $e_{p}(x) \propto\left[1-\cos \left(2 \pi x / a_{0}\right)\right]$, and the corresponding force takes the form $f_{p}(x) \approx-f_{0} \sin \left(2 \pi x / a_{0}\right)$. Analyzing the characteristic lengths for the present situation, one finds that (i) the position $x_{m}$ of steepest slope in $f_{p}$ coincides with $a_{0} / 2$, (ii) $x_{0 \mathrm{~L}}=x_{m}-f_{p}\left(x_{m}\right) / \bar{C}=x_{m}$ because $f_{p}\left(x_{m}\right)=0$, and (iii) $x_{0}(T)=x_{0 \mathrm{~L}}$ because of symmetry arguments. Furthermore, the branch edges $\pm x_{-}$have disappeared and those at $\pm x_{+}$overlap with the next period, i.e., $\left|x_{+}\right|>a_{0} / 2$, see Fig. 6. As a result, in a zero-field-cooled sample, the high-field limit of the Campbell length $\lambda_{\mathrm{C}}$ is always determined by the (single) jump at $a_{0} / 2, \Delta f_{\text {pin }}=\left.\Delta f_{\text {pin }}\right|_{a_{0} / 2}$; in analogy to our previous nomenclature, we call this the case (a') and note that this reversible (non-hysteretic) behavior becomes the generic case at high fields. In the zero-field-cooled (or Bean critical) state, the penetration depth $\lambda_{\mathrm{C}}$ involves the (slightly smaller) jump at $x_{+}, \Delta f_{\text {pin }}=\left.\Delta f_{\text {pin }}\right|_{x_{+}}$, and hence $\left.\left.\lambda_{\mathrm{C}}\right|_{\mathrm{FC}} \lesssim \lambda_{\mathrm{C}}\right|_{\mathrm{ZFC}}$.

Again, simple closed-form expressions can be found at small values of $\kappa \gtrsim 1$. Near the Labusch point, the results Eqs. (39) and (40) remain valid [with $\gamma=\left(4 \pi^{3} / a_{0}^{3}\right) f_{0}$ and $x_{0 \mathrm{~L}} \rightarrow a_{0} / 2$, from which expressions for $\lambda_{\mathrm{C}}$ follow immediately. At large $\kappa$, the low-field result $\Delta f_{\text {pin }} \simeq \bar{C} \kappa \xi$ is cut off by the lattice period when $\kappa \xi>a_{0}$ and hence $\Delta f_{\text {pin }} \simeq \bar{C} a_{0}$, resulting in a Campbell length

$$
\lambda_{\mathrm{C}}^{2} \simeq \frac{B_{0} \Phi_{0}}{4 \pi n_{p} t_{\perp}} \frac{1}{\bar{C} a_{0}} \sim \frac{\lambda^{2}}{n_{p} a_{0} \xi^{2}} \frac{\xi}{a_{0}},
$$

a factor $\kappa \xi / a_{0}$ larger than the low-field result (43). However, for such strong pinning the applicability of the elastic theory becomes questionable. Indeed, as suggested by numerical analysis ${ }^{36137}$, the elastic theory might break down due to plastic instabilities of the vortex lattice. It has been shown ${ }^{36}$ that a vortex detaches from an infinitely strong pinning center via loop formation and subsequent vortex cutting and reconnection, which is a highly non-elastic process. Similarly, a computational study based on a time-dependent GinzburgLandau solver ${ }^{4 / 37}$ has demonstrated that small pinning centers are not capable of holding multiple vortices at the same time. Indeed, rather than trapping a second flux line, the defect trades one vortex for the next, with the first vortex pushed out of the pinning well by the following one. These insights suggest that the maximal pinning distance $x_{+}$should be limited to $a_{0}$, producing a force jump $\Delta f_{\text {pin }} \simeq \bar{C} a_{0}$. This result coincides with the one above and the Campbell length is still given by Eq. (44). The situation is more subtle when considering the value of $j_{c}$ at high fields and strong pinning. Assuming the elastic theory to remain valid, one obtains a jump in energy $\Delta e_{\text {pin }}=f_{0} a_{0}$ and $j_{c}$ is reduced by a factor $a_{0} / \kappa \xi<1$ as compared to the low-field result (27) (note that multiply overlapping pinned branches appear when $\left.\kappa \xi>a_{0}\right)$. Accounting for plasticity, the jump in energy is even further reduced, $\Delta e_{\text {pin }} \approx \bar{C} a_{0}^{2} / 2$, and the critical current takes the universal form $j_{c} \sim j_{\mathrm{dp}}\left(n_{p} a_{0} \xi^{2}\right)$, a factor $\left(a_{0} / \kappa \xi\right)^{2}$ smaller than the low-field result $(27)$.

For mid-range magnetic fields, neither the singlevortex nor the sinusoidal force profile is accurate. Starting from the limit of high fields, in addition to the basic sinusoidal force profile, further higher-order harmonics need to be taken into account. As the field is sufficiently lowered, the position $x_{m}$ of the maximal slope in the (bare) force profile detaches from $a_{0} / 2$, and a second (unpinned) branch develops.

\section{Comparison between different regimes}

It is interesting to analyze the scaling behavior of the Campbell length as a function of the strong pinning parameter $\kappa$. Indeed, when expressing $\lambda_{\mathrm{C}}^{2}$ [Eq. [28)] in units of $\lambda^{2} / \nu_{p}$, with $\nu_{p}=n_{p} a_{0} \xi^{2}$ the dimensionless small density parameter, we find

$$
\nu_{p} \frac{\lambda_{\mathrm{C}}^{2}}{\lambda^{2}} \sim \frac{\bar{C} \xi}{\Delta f_{\text {pin }}} \frac{\xi}{t_{\perp}}
$$

where we have used that $\bar{C} \sim \varepsilon_{0} / a_{0}$, see Sec. III A. When pushing the system across the Labusch point $\kappa=1$ into 
the strong pinning regime, we find a universal scaling [see Eqs. [39) and (40)]

$$
\nu_{p} \frac{\lambda_{\mathrm{C}}^{2}}{\lambda^{2}} \sim \frac{1}{\sqrt{\kappa-1}},
$$

which is valid at all fields and for different vortex states (FC as well as ZFC), see Fig. 7. Combining this result with the standard scaling 22 of the critical current density $j_{c} \sim j_{\mathrm{dp}} \nu_{p}\left(\xi / a_{0}\right)^{2}(\kappa-1)^{2}$ in the vicinity of the Labusch point, we arrive at the relation $j_{c} \sim\left(c \xi B_{0} / \lambda_{\mathrm{C}}\right)(\kappa-1)^{3 / 2}$ which strongly differs from the scaling $j_{c} \sim c \xi B_{0} / \lambda_{\mathrm{C}}$ obtained within a phenomenological approach. At intermediate values of $\kappa$, we can write $\Delta f_{\text {pin }} \sim \bar{C} x_{\text {jp }}$ with $x_{\mathrm{jp}}=x_{-}, x_{0 \mathrm{~L}} \simeq \xi, x_{+}$for the cases FC (b), FC (a), and ZFC/FC (b'). For the cases FC (b) and (a) the further change in $\nu_{p} \lambda_{\mathrm{C}}^{2} / \lambda^{2}$ is small, $\propto \xi / t_{\perp}$ and $\propto \xi^{2} / t_{\perp} x_{-}$, respectively, while a pronounced decrease appears for the ZFC and FC (b') cases, $\propto \xi / \kappa t_{\perp}$. At large $\kappa \gg 1$, the quantities $x_{-}, x_{+}, t_{\perp}$ saturate as they reach the scale $a_{0}$, with a corresponding change in the expression for $\nu_{p} \lambda_{\mathrm{C}}^{2} / \lambda^{2}$. Finally, case FC (b') assumes that $x_{+} \simeq \kappa \xi$ decreases with increasing $\kappa$ and naturally terminates when this condition is violated. The scaling behavior of $\nu_{p} \lambda_{\mathrm{C}}^{2} / \lambda^{2}$ and the appearance of hysteretic behavior upon reheating is illustrated in Fig. 7. The low-field scaling discussed above changes over to the high-field behavior (see section III C 3, $\nu_{p} \lambda_{\mathrm{C}}^{2} / \lambda^{2} \propto 1 / \sqrt{\kappa-1}, 1$ at small and large $\kappa>1$, respectively) when the intervortex distance $a_{0}$ approaches $\xi$. These results can be used to characterize the pinscape by combining theoretical input on $\kappa(T, H)$ for various pinning models (see below) with experimental data for $\lambda_{\mathrm{C}}$. Such information is of great value when simulating vortex dynamics within a numerical approach, e.g., using time-dependent Ginzburg-Landau theory 4 .

\section{PINNING MODELS}

In order to proceed further towards quantitative predictions of the Campbell response, we have to specify all relevant quantities entering the pinning problem. In particular, the temperature- and field-dependence of the vortex elasticity $\bar{C}$ and the pinscape energy $e_{p}$ have to be determined. This is the central topic of this Section and will allow us later to follow the evolution of the effective force $f_{\text {pin }}$ upon cooling and reheating the system in the field as typically done in an experiment. Here, we focus on the comparison between different pinning models and defer the comparison to experiments to Sec. V.

The $T$ - and $H$-dependence of the effective elasticity $\bar{C}$ and of the pinning energy $e_{p}$ is mainly determined by the Ginzburg-Landau parameters $\lambda$ and $\xi$, with the superfluid density $n_{s} \propto \lambda^{-2}$ scaling both with temperature and field, $\lambda^{-2} \simeq \lambda_{0}^{-2}\left(1-T / T_{c}\right)\left[1-B / H_{c 2}(T)\right] \simeq \lambda_{0}^{-2}(1-$ $\tau-b_{0}$ ), while the coherence length scales with temperature only, $\xi^{-2}(T)=\xi_{0}^{-2}(1-\tau)$. Here we have used the scaling of the upper critical field $H_{c 2}(T)=\Phi_{0} / 2 \pi \xi^{2}(T)$

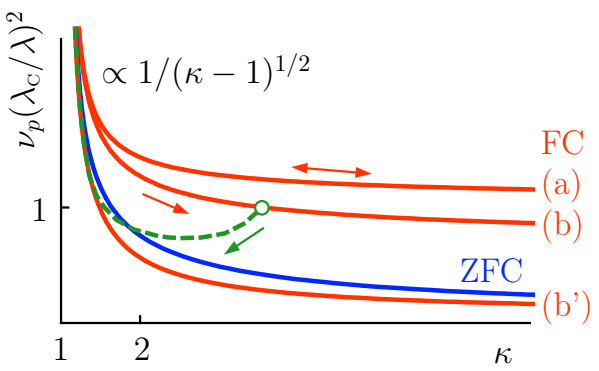

FIG. 7. Schematic view of the $\kappa$-dependence of the parameter $\nu_{p}\left(\lambda_{\mathrm{C}} / \lambda\right)^{2}$, with $\nu_{p}=n_{p} a_{0} \xi^{2} \ll 1$ the small dimensionless density parameter. Upon crossing the Labusch point $\kappa=1$ and entering the strong pinning regime, all curves first decrease as $1 /(\kappa-1)^{1 / 2}$. Subsequently, the cases FC (a) and $\mathrm{FC}$ (b) decay weakly $\propto \xi / t_{\perp}$ and $\propto \xi^{2} / t_{\perp} x_{-}$, respectively. The cases ZFC and FC (b') decay more rapidly $\propto \xi / \kappa t_{\perp}$. At large $\kappa$, the decrease slows down when $x_{+} \simeq \kappa \xi$ and $x_{-}$reach the scale $a_{0}$; note that $t_{\perp}=2 x_{-}$within our analysis. The field-cooled cases (b) and (b') exhibit a hysteretic response upon reversing the direction of $\kappa$ (see dashed line). The hysteresis loop shown for case FC (b) merges with the curve FC (b') when taking $\kappa$ back to unity. Similarly, reversing $\kappa$ on curve FC (b') one approaches the curve FC (b) (not shown).

and have introduced the reduced temperature $\tau=T / T_{c}$ and the reduced field $b_{0}=B_{0} / H_{c 2}(0)$. Note that the superfluid density vanishes on approaching $H_{c 2}(T)$ where $1-\tau-b_{0}=0$. In this section, we shall not burden our expressions with the more complicated details of the $H$ - and $T$-dependence in the superconducting phase but rather extrapolate the Ginzburg-Landau scaling valid near $T_{c}$ to the entire phase diagram. In Sec. $\mathrm{V}$, where we confront our predictions with experimental data, a more accurate scaling will be chosen.

The Labusch parameter is given through the ratio of pinning curvature and elasticity, $\kappa=\max _{x}\left[-e_{p}^{\prime \prime}(x)\right] / \bar{C}$. For small defects, we approximate the curvature $-e_{p}^{\prime \prime} \approx$ $e_{0} V_{\text {pin }} / \xi^{2}$, with $e_{0}$ the typical gain in energy density and $V_{\text {pin }}$ the relevant pinning volume, hence

$$
\kappa \approx \frac{e_{0} V_{\text {pin }}}{\xi^{2} \bar{C}} .
$$

In the following, we consider four different pinning models (metallic and insulating inclusions, as well as $\delta T_{c^{-}}$, or $\delta \ell$-pinning) and evaluate the behavior of $\kappa$ within the $H-T$ phase diagram.

\section{A. Elasticity $\bar{C}$}

We first evaluate the effective elasticity $\bar{C}$, a quantity that is independent of the chosen pinning model. Starting from its definition (14) and the subsequent discussion, the effective elasticity $\bar{C}=\left(\nu a_{0}^{2} / \lambda\right)\left[c_{66} c_{44}(\mathbf{0}, 0)\right]^{1 / 2}$ involves the non-dispersive shear $\left(c_{66}\right)$ and the bulk tilt $\left[c_{44}(0)\right]$ moduli as well as the London penetration depth $\lambda$. Inserting the standard expressions ${ }^{30138}$ for the elastic 
moduli

$$
c_{44}(\mathbf{0}, 0)=\frac{B_{0}^{2}}{4 \pi} \quad \text { and } \quad c_{66} \simeq \frac{\varepsilon_{0}}{4 a_{0}^{2}}\left[1-\frac{B_{0}}{H_{c 2}(T)}\right]
$$

and using the scaling $\varepsilon_{0} \propto(1-\tau-b)$ while $c_{66} \propto(1-$ $\left.B_{0} / H_{c 2}\right)^{2}\left(1-T / T_{c}\right)=(1-\tau-b)^{2} /(1-\tau)$ we find that

$$
\bar{C}=\nu\left(\frac{\Phi_{0}}{4 \pi \lambda_{0}}\right)^{2} \sqrt{\frac{b_{0}}{2 \xi_{0}^{2}}} \frac{\left(1-\tau-b_{0}\right)^{3 / 2}}{(1-\tau)^{1 / 2}} .
$$

The factor $\left(1-\tau-b_{0}\right)^{3 / 2}$ describes the softening of the lattice near the $H_{c 2}$-line.

\section{B. Small defects}

We consider a defect in the form of a small inclusion of radius $\rho \ll \xi$. A vortex placed a distance $x$ away from this pin will experience an energy decrease

$$
e_{p}(x)=-\int d x^{\prime} e_{0}\left(x^{\prime}\right)\left[1-\left|\psi_{0}\left(x-x^{\prime}\right)\right|^{2}\right] .
$$

The shape of the vortex solution $\psi_{0}(x)$ can be obtained within Ginzburg-Landau (GL) theory 39 ; at low fields, it is well described by the expression $\frac{40 \mid 41}{\mid}\left|\psi_{0}(x)\right|^{2}=$ $x^{2} /\left(x^{2}+2 \xi^{2}\right)$, producing a Lorentzian shape for the pinning potential

$$
e_{p}(x)=-\frac{e_{0} V_{\text {pin }}}{1+x^{2} / 2 \xi^{2}} .
$$

In the high field limit $1-B_{0} / H_{c 2} \ll 1$, we approximate the vortex solution ${ }^{42}$ by the one-dimensional harmonic

$$
\left|\psi_{0}(x)\right|^{2}=\frac{1}{2}\left[1-\cos \left(2 \pi x / a_{0}\right)\right]
$$

and evaluating Eq. (50), we arrive at a periodic pinning profile

$$
e_{p}(x)=-\frac{e_{0} V_{\text {pin }}}{2}\left[1+\cos \left(2 \pi x / a_{0}\right)\right]
$$

\section{Insulating defect}

For an insulating inclusion, the typical energy that a vortex state gains by aligning a flux line with the defect is determined by the condensation energy density and the pin volume. The former derives from a minimization of the Ginzburg-Landav ${ }^{39}$ (GL) functional $f_{\mathrm{GL}}=\alpha|\psi|^{2}+\beta|\psi|^{4} / 2$, providing an order parameter $\left|\Psi_{0}\right|^{2}=|\alpha| / \beta$; inserting this back to $f_{\mathrm{GL}}$, we obtain $e_{0}=-|\alpha|^{2} / 2 \beta$. While in standard GL theory the temperature dependence derives from $\alpha(T)=\alpha_{0}\left(T-T_{c}\right)=$ $-\alpha_{0} T_{c}(1-\tau)$, close to the $H_{c 2}(T)$-line we can adopt a lowest Landau level approximation of the GL functiona ${ }^{43}$ by
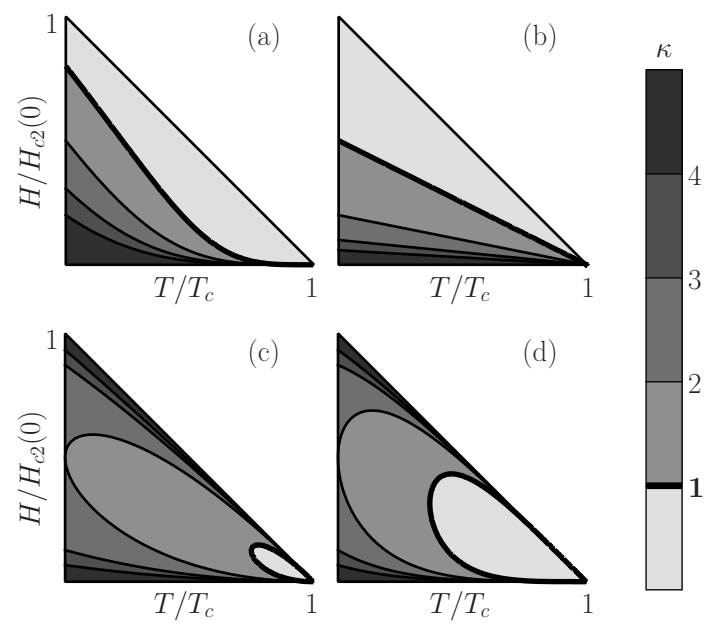

FIG. 8. Generic density plot of the strong pinning parameter $\kappa(T, H)$ for four different pinning models. The Labusch criterion $\kappa=1$ determines the transition line (bold), where the pinscape changes from weak $(\kappa<1)$ to strong $(\kappa>1)$ pinning. Depending on the parameters, this separatrix assumes a different shape and location in the diagram. For insulating (a) and metallic (b) inclusions the pinscape is weak upon crossing $H_{c 2}(T)$ and turns strong at low fields/temperatures, see Eqs. (55) and (56), respectively. For defects inducing a local change in $T_{c}(\mathrm{c})$ or in the mean free path $\ell$ [via a local change of the effective mass, panel (d)] the pinscape is strong upon entering the superconducting phase, see Eqs. (58) and (61), respectively. For a good visibility of all the features, we have used the parameters $\rho^{3}=2 \xi_{0}^{3}$ and $\delta T_{c} / T_{c}=\delta \ell / \ell=0.5$.

replacing this temperature dependence with a temperature and field dependence $\alpha(T, B)=-\alpha_{0} T_{c}\left(1-\tau-b_{0}\right)$ (the order parameter then assumes the role of the amplitude of the space-modulated solution). The combination with the expression for the (zero-temperature) thermodynamic critical field $H_{c 0}^{2} / 4 \pi=\alpha_{0}^{2} T_{c}^{2} / \beta$ then produces an overall temperature and field scaling of the condensation energy of the form

$$
e_{0}\left(T, B_{0}\right) \approx \frac{H_{c 0}^{2}}{8 \pi}\left(1-\tau-b_{0}\right)^{2},
$$

in agreement with the discussion in 44. Combining this result with the effective elasticiy $\bar{C}$ in Eq. $\overline{49}$, we make use of Eq. 477) to find the following explicit dependence on $b_{0}$ and $\tau$ of the strong pinning parameter,

$$
\kappa \approx \frac{\rho^{3}}{\xi_{0}^{3}} \sqrt{\frac{1}{b_{0}}}(1-\tau)^{3 / 2}\left(1-\tau-b_{0}\right)^{1 / 2} .
$$

For insulating pins the strong pinning parameter reveals two important asymptotic regimes, see Fig. 8(a). First, $\kappa$ vanishes along the entire $H_{c 2}$-line defined through $1-$ $\tau-b_{0}=0$. As a consequence, the insulating defects act as weak pins upon crossing $H_{c 2}(T)$. Second, $\kappa$ grows as $b_{0}^{-1 / 2}$ at low fields (a consequence of the softening of $\bar{C}$ at low fields), thus guaranteeing that the defect turns into a strong pinning center with $\kappa>1$. 


\section{Metallic defect}

Similar happens in the case of a metallic defect which affects the superconductor via the proximity effect. For a metallic inclusion, the order parameter is substantially suppressed within a volume $\sim \xi^{3}$ around the pin. This provides the possibility for the flux line lattice to gain the condensation energy density (54) over a larger volume $V_{\text {pin }} \rightarrow V_{\text {pin }}^{\text {eff }} \approx(4 \pi / 3) \xi^{3}$. In this case, the real size of the defect drops out of the final result and the strong pinning parameter

$$
\kappa \approx \sqrt{\frac{1}{b_{0}}}\left(1-\tau-b_{0}\right)^{1 / 2}
$$

shows a qualitatively similar behavior as the insulating pin in Eq. (55), see Fig. 8(b): the metallic pins are weak upon entering the superconducting phase when traversing the $H_{c 2}$-line and they turn strong at low temperatures and fields where $\kappa \approx b_{0}^{-1 / 2}$.

\section{E. $\delta T_{c}$-pinning}

A very different pinning behavior is observed when defects locally change the critical temperature, $T_{c} \rightarrow$ $T_{c}-\delta T_{c}$. Such a local variation in $T_{c}$ has to be included in the quadratic term of the GL energy functional and adds a correction $\alpha_{0} \delta T_{c}|\psi|^{2}$. Making use of the above results for $|\psi|^{2}$ and $H_{c 0}^{2}$, we arrive at the following expression for the local energy gain

$$
e_{0}\left(T, B_{0}\right) \approx \frac{H_{c 0}^{2}}{4 \pi} \frac{\delta T_{c}}{T_{c}}\left(1-\tau-b_{0}\right) .
$$

The combination with the expression $(49)$ for the elasticity $\bar{C}$ provides the scaling for $\kappa$ in the form

$$
\kappa \approx \frac{\rho^{3}}{\xi_{0}^{3}} \frac{\delta T_{c}}{T_{c}} \sqrt{\frac{1}{b_{0}}}(1-\tau)^{3 / 2}\left(1-\tau-b_{0}\right)^{-1 / 2} .
$$

As a result, $\kappa$ is divergent both at low fields $b_{0} \rightarrow 0$ and near the $H_{c 2}(T)$-line. On the other hand, the factor $(1-\tau)^{3 / 2}$ suppresses $\kappa$ near $T_{c}$. This leads to a peculiar weak-pinning lobe extending from $\left(T_{c}, H=0\right)$ into the phase diagram, see Fig. 8 (c). Lowering the temperature at a constant field (horizontal cut) or changing the field at a constant temperature (vertical cut), the system might cross the Labusch point twice, changing from strong to weak and back to strong pinning. As observed by Larkin and Ovchinnikov 21 , the strengthening of pinning near $H_{c 2}(T)$ manifests itself in a sharp increase of the critical current, a feature known as peak effect $\frac{45 \mid 46}{\text {. }}$.

\section{F. $\delta \ell$-pinning}

Finally, we address the pinning due to local changes of the mean free path, $\ell \rightarrow \ell-\delta \ell$. The dependence of the Ginzburg-Landau functional on the mean free path appears in the gradient term $\left(\hbar^{2} / 2 m\right)|\nabla \psi|^{2}$. Indeed, a microscopic calculation $\frac{47}{47}$ provides the additional factor $\chi\left(\rho_{\ell}\right), \rho_{\ell}=\hbar v_{\mathrm{F}} / 2 \pi T_{c} \ell \simeq \xi_{0} / \ell$, with $\chi \approx 1$ and $\chi \approx\left[\pi^{2} / 7 \zeta(3)\right] / \rho_{\ell}$ in the clean and dirty limits, respectively. As a result, the coherence length $\xi(T)$ depends on disorder via

$$
\xi^{2} \approx \xi_{0}^{2} \chi\left(\rho_{\ell}\right) /(1-\tau)
$$

with $\xi_{0}$ the $T=0$ clean-limit coherence length. The (quenched) fluctuations in $\ell$ translate into fluctuations in the gradient term and entail a change in the energy density $e_{0}$ of the form

$$
e_{0}\left(T, B_{0}\right) \approx \frac{H_{c 0}^{2}}{4 \pi} \frac{\delta \ell}{\ell}(1-\tau)\left(1-\tau-b_{0}\right) .
$$

We then arrive at a Labusch parameter in the form

$$
\kappa \approx \frac{\rho^{3}}{\xi_{0}^{3}} \frac{\delta \ell}{\ell} \sqrt{\frac{1}{b_{0}}}(1-\tau)^{5 / 2}\left(1-\tau-b_{0}\right)^{-1 / 2},
$$

exhibiting a qualitative similar behavior as the one found for $\delta T_{c}$-pinning but with a larger exponent $5 / 2$ for the $(1-\tau)$ factor $\left(3 / 2\right.$ for $\delta T_{c}$-pinning), pushing the weakpinning lobe deeper into the phase diagram, see Fig. $8(d)$.

\section{COMPARISON TO EXPERIMENTS}

Equipped with a microscopic expression for the Campbell penetration depth, we discuss experimental signatures that provide strong support for our new results. Below, we focus on few original studies by Campbell 617 and Lowell $\frac{8 / 48}{4}$ as well as more recent studies by Prozorov and co-workers, see Refs. [17], [20], and [49].

\section{A. General comparison}

We have identified four major experimental signatures that find a natural explanation within our analysis of $a c$ magnetic response.

a. Low versus high dc fields. In early work, e.g., by Campbell ${ }^{6}$ or Lowell $\stackrel{8}{ }$, it has been noted that the $a c$ magnetic response does not depend on the state preparation (field-cooled or zero-field-cooled). A simple (piece-wise linear) force model was put forward ${ }^{7 / 48}$ in support of this result. The dependence of the $a c$ magnetic penetration depth $\lambda_{\mathrm{C}}$ on the vortex state preparation was first reported by Prozorov and co-worker\$17. In recent years, different Campbell lengths for the field-cooled and zerofield-cooled states have been observed ${ }^{2049}$ in a wide range of materials, including Niobium, $\mathrm{MgCNi}_{3}, \mathrm{SrPd}_{2} \mathrm{Ge}_{2}$, the high-temperature superconductor $\mathrm{Bi}_{2} \mathrm{Sr}_{2} \mathrm{CaCu}_{2} \mathrm{O}_{8}$, $\mathrm{Pr}_{1-x} \mathrm{Ce}_{x} \mathrm{CuO}_{4}$, and the organic superconductor $\beta^{\prime \prime}$ (ET) ${ }_{2} \mathrm{SF}_{5} \mathrm{CH}_{2} \mathrm{CF}_{2} \mathrm{SO}_{3}$. The new results provided by our strong pinning analysis are compatible with both types of 
observations: at high $d c$ fields, the typical setup of early experiments, the Campbell lengths are (almost) identical, see Sec. III C 3, while they are different (sometimes even parametrically) at low fields, see sections III C 1 and III C 2.

b. Finite $\lambda_{\mathrm{C}}$ in the critical state. The phenomenological theory, on which the interpretation of most $a c$ experiments has been based so far, predicts 17 a divergent Campbell length for the zero-field-cooled state, $\lambda_{\mathrm{C}} \propto\left(1-j / j_{c}\right)^{-1 / 4}$, as the curvature $\alpha(j) \propto\left(j_{c}-j\right)^{1 / 2}$ of the pinning well vanishes on approaching the critical state. Not only is the experimentally observed Campbell length in the Bean critical state finite, but in some materials it is even smaller than that of the field-cooled state, $\left.\lambda_{\mathrm{C}}\right|_{\mathrm{ZFC}}<\left.\lambda_{\mathrm{C}}\right|_{\mathrm{FC}}$. Both features are well understood within the strong pinning framework. The Campbell length $\lambda_{\mathrm{C}}$ results from an averaging of the local curvature which can (depending on the pinning parameters) get reduced when changing the branch occupation from the field-cooled state to the zero-field-cooled state. In the latter situation, the application of an $a c$ field will first generate flux pulses that penetrate the sample and change the $d c$ field inside the material26. At the end of this transient initialization, the response of the vortex system is perfectly regular and characterized by a finite Campbell length $\lambda_{\mathrm{C}}$.

c. Hysteresis upon thermal cycling of $\left.\lambda_{\mathrm{C}}\right|_{\mathrm{FC}}$. The strong pinning framework of ac magnetic response predicts the appearance of hysteretic Campbell lengths for the field-cooled samples upon thermal cycling. Such hysteretic behavior has been observed in experiments by Prozorov and co-workers, see Ref. 25].

d. Universality of $\left.\lambda_{\mathrm{C}}\right|_{\mathrm{ZFC}}$ for different critical states. Within our microscopic analysis, the direction of the Lorentz force $\pm j_{c}$ does not affect the asymptotic (i.e., large times $t \gg 2 \pi / \omega)$ oscillatory response of the vortex lattice in the critical state. Hence, the Campbell length is independent on whether the external field $H$ is reached from below (ramping up) or from above (ramping down). This independence has been experimentally demonstrated 17 . We note that the transient behavior before reaching the asymptotic periodic regime may exhibit differences between the two ramping directions, as an opposite $d c$-shift is expected when ramping the field down to $H$, with the number of cycles needed to reach the asymptotic behavior depending on the depth of the critical state. This prediction could be verified in an experiment.

\section{B. Comparison to $\mathrm{SrPd}_{2} \mathrm{Ge}_{2}$}

Finally, we provide a semi-quantitative comparison of our microscopic analysis of the Campbell length $\lambda_{\mathrm{C}}$ with measurements on a single-crystal germanide superconductor $\mathrm{SrPd}_{2} \mathrm{Ge}_{2}$ with $T_{c}=2.7 \mathrm{~K}$ and $H_{c 2}=0.49 \mathrm{~T}$. Vortex pinning in this ternary compound, parent to the iron- and nickel-pnictides, is likely to be strong. ${ }^{20 \mid 50}$ Its $a c$
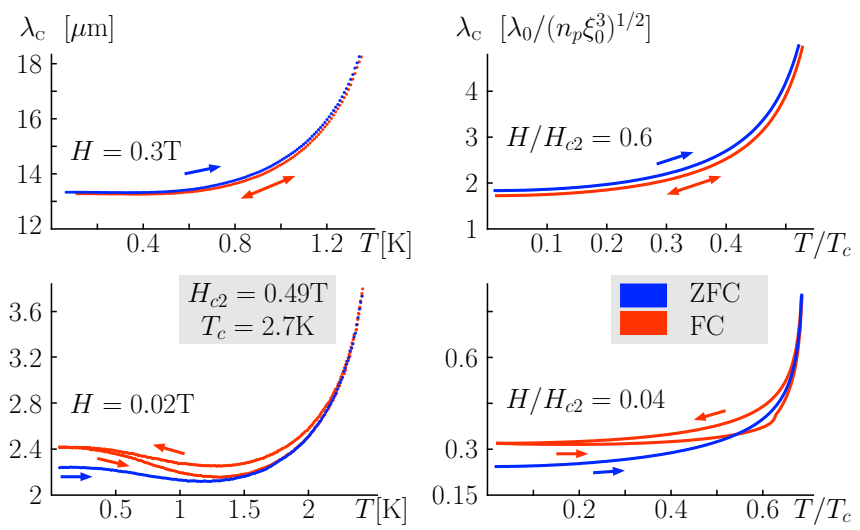

FIG. 9. Comparison between the Campbell length obtained from experiments (left) and from numerics (right). On the experimental side, we show representative traces for high $\left(H=0.3 \mathrm{~T} \approx 0.6 H_{c 2}\right)$ and low fields $\left(H=0.02 \mathrm{~T} \approx 0.04 H_{c 2}\right)$, see Ref. [20] and 25]. The corresponding theoretical curves are obtained from (i) solving Eq. 21) numerically for an insulating pin (see Sec. IV C), (ii) determining the relevant jumps in the force profile, and (iii) evaluating $\lambda_{\mathrm{C}}$ through Eq. 28. The sharp upturn appearing at $T \approx 0.65 T_{c}$ upon reheating the field-cooled state in low fields is due to the change in the jump position $x_{\mathrm{jp}}$ as it reaches the branch edge $x_{+}$(corresponding to $T_{2}$ in Fig. 5 .

response has been investigated with a tunnel-diode technique in Ref. 20]. We focus on two traces of $\lambda_{\mathrm{C}}$ recorded at different applied $d c$ fields $0.02 \mathrm{~T}$ and $0.3 \mathrm{~T}$ and taken from Fig. 4(a) of Ref. 20]. In Fig. 9 (left) we show an enlarged view of these traces, with the zero-field-cooled data in blue and the thermally cycled field-cooled data (see arrows for the temperature direction) in red. At high fields $0.3 \mathrm{~T} \approx 0.6 H_{c 2}$, the Campbell lengths are almost identical, but with $\left.\lambda_{\mathrm{C}}\right|_{\mathrm{ZFC}}$ slightly larger than $\left.\lambda_{\mathrm{C}}\right|_{\mathrm{FC}}$. The low-field trace at $0.02 \mathrm{~T} \approx 0.04 H_{c 2}$ is much richer: the field-cooled and zero-field-cooled Campbell lengths clearly differ from each other. Moreover, the Campbell length of the field-cooled state shows a strong hysteresis upon thermal cycling. The heating branch (arrow to the right) deviates from the cooling branch and approaches the zero-field-cooled Campbell length at higher temperatures. Finally all Campbell length curves feature a minimum at around $1.2 \mathrm{~K}$.

The theoretical analysis of $\lambda_{\mathrm{C}}$, see right of Fig. 9, requires the knowledge of the pinscape $e_{p}(x)$ for all fields $B_{0}$. We interpolate between the two limits of low [Eq. (51)] and high [Eq. (53)] fields by periodically summing the low-field profile,

$$
\chi(x)=\sum_{n=-\infty}^{\infty} \frac{1}{1+\left(x+n a_{0}\right)^{2} / 2 \xi^{2}}
$$

and applying the normalization (neglected in Ref. [25])

$$
e_{p}(x)=e_{0} V_{\text {pin }} \frac{\chi(x)-\chi(0)}{\chi\left(a_{0} / 2\right)-\chi(0)} .
$$


For a better comparison to experiment, we replace the simple Ginzburg-Landau scaling $1-\tau$ used in Sec. IV] by the more accurate two-fluid-model scaling $1-\tau^{2}$; the latter properly captures the saturation of the phenomenological parameters at low temperatures. In order to find the temperature and field dependence of $\lambda_{\mathrm{C}}$, we numerically evaluate the force profile $f_{\text {pin }}(x)$ and the relevant jumps $\Delta f_{\text {pin }}$ for the zero-field-cooled and field-cooled situations. For the latter, we make use of a numerical routine that follows the branch evolution and the associated occupation upon lowering the system's temperature and its subsequent heating.

The quantity $\left(n_{p} \xi_{0}^{3}\right)^{1 / 2} \lambda_{\mathrm{C}} / \lambda_{0}$ solely depends on the ratio $\rho / \xi_{0}$, with $\rho$ the radius of the insulating defect. This parameter governs the extent of the strong pinning region within the $H$ - $T$ diagram. It turns out, that choosing an insulating defect of radius $\rho \approx 1.82 \xi_{0}\left(\rho \approx 1.6 \xi_{0}\right.$ in Ref. [25]), see Eq. (54), provides a good description of the experimental data. Indeed, the results shown on the right of Fig. 9 reproduce all relevant features of the experiment: These are the closeness between the ZFC and FC Campbell lengths at high fields, as well as their sequence in magnitude, $\left.\lambda_{\mathrm{C}}\right|_{\mathrm{ZFC}}>\left.\lambda_{\mathrm{C}}\right|_{\mathrm{FC}}$, as predicted by point $b$ in Sec. $\mathrm{VA}$. At low fields, the sequence in magnitude changes $\left.\lambda_{\mathrm{C}}\right|_{\mathrm{ZFC}}<\left.\lambda_{\mathrm{C}}\right|_{\mathrm{FC}}$ (see point $c$ in Sec. VA and the field-cooled Campbell length turns hysteretic (point $d$ in Sec. V A.

Making use of the phenomenological parameters characterizing the germanide superconductor ${ }^{20}, \lambda_{0}=426 \mathrm{~nm}$ and $\xi_{0}=25 \mathrm{~nm}$, we find that a defect density $n_{p} \sim$ $10^{14} \mathrm{~cm}^{-3}$ (corresponding to a distance between defects of order $10 \xi_{0}$ ) provides the correct magnitude of $\lambda_{\mathrm{C}}$ and is consistent with the small density condition $n_{p} a_{0} \xi^{2} \ll 1$.

\section{CONCLUSION}

We have investigated the linear ac magnetic response of type-II superconductors in the Shubnikov phase as characterized through the Campbell length $\lambda_{\mathrm{C}}$, the penetration depth of the $a c$ signal. Starting from the microscopic theory of strong pinning, we have shown that the Campbell length involves specific jumps in the (multivalued) pinning force profile corresponding to abrupt changes in the occupation of the force branches. With this new tool at hand, we have discussed the generic behavior of the Campbell length (i) near and away from the Labusch point describing the onset of strong pinning, (ii) at low and high magnetic fields, and (iii) for both field-cooled (FC) and zero-field-cooled (ZFC) state preparations. Several new features have been observed: first, the FC and ZFC states probe different force jumps and hence result in (possibly even parametrically) different Campbell lengths. Second, in the critical state, a transient initialization changes the $d c$ field by $h_{a c}$ after which the response follows a regular $a c$ dynamics with a finite Campbell length. Third, for the field-cooled state, we predict a possible hysteretic response of the Campbell length upon thermal cycling. On the road towards quantitative predictions, we have studied the scaling behavior of four types of defects (insulating and metallic inclusions, $\delta T_{c^{-}}$and $\delta \ell$-pinning) and have constructed $H-T$ scaling diagrams for the pinning strength $\kappa$. Finally, we have confronted our theory with experimental data and have found good qualitative and even semi-quantitative agreement.

The framework presented here provides a quantitative relation between the macroscopic Campbell length $\lambda_{\mathrm{C}}$ and the underlying microscopic pinning landscape. The power of this approach lies in the ability to distinguish between different vortex configurations, e.g., field-cooledand zero-field-cooled states or an arbitrary state 'inbetween', allowing for a spectroscopic analysis of the pinscape. While the measurement of $\lambda_{\mathrm{C}}$ combined with theoretical insights provides access to bulk averaged parameters of the pinscape such as the defects' nature (insulating, metallic, $\delta T_{c^{-}}$or $\delta \ell$-type), its density $n_{p}$, and shape $e_{p}(\boldsymbol{r})$, recent experiments using scanning STM and scanning SQUID techniques allow for space-resolved imaging of the pinscape $e^{23 \mid 24}$. Together, these novel techniques provide valuable input for advanced numerical simulations of (driven) vortex matter, e.g., based on (timedependent) Ginzburg-Landau theory ${ }^{45}$. The outcome of such simulations may then be used to better understand the signatures observed in experiments 51 , thus closing the loop in a fruitful comparison of theory, experiment, and numerical simulation. In future work it will be interesting to uncover other types of experimental signatures providing further information on the strong pinning landscape.

\section{ACKNOWLEDGMENTS}

We acknowledge financial support of the Swiss National Science Foundation (SNSF) through the NCCR MaNEP. The authors are indebted to Ruslan Prozorov for providing us with the experimental data presented in Fig. 9 .
1 L. V. Shubnikov, V. I. Khotkevich, Y. D. Shepelev, and Y. N. Riabinin, [Zh. Eksp. Teor. Fiz. 7, 221 (1937)] Ukrainian Journal of Physics 53, 42 (2008).

2 A. A. Abrikosov, [Zh. Eksp. Teor. Fiz. 32, 1442 (1957)] JETP 5, 1174 (1957).
3 J. Bardeen and M. J. Stephen, Physical Review 140, A1197 (1965).

4 I. A. Sadovskyy, A. E. Koshelev, C. L. Phillips, D. A. Karpeev, and A. Glatz, Journal of Computational Physics 294, 639 (2015). 
${ }^{5}$ I. A. Sadovskyy et al., unpublished (2015).

6 A. M. Campbell, Journal of Physics C: Solid State Physics 2, 1492 (1969).

7 A. M. Campbell, Journal of Physics C: Solid State Physics 4, 3186 (1971).

8 J. Lowell, Journal of Physics F: Metal Physics 2, 547 (1972).

9 A. M. Campbell, Philosophical Magazine Part B 37, 149 (1978).

10 J. G. Bednorz and K. A. Müller, Zeitschrift für Physik B 64, 189 (1986).

11 E. H. Brandt, Physical Review Letters 67, 2219 (1991).

12 A. E. Koshelev and V. M. Vinokur, Physica C 173, 465 (1991).

13 M. W. Coffey and J. R. Clem, Physical Review Letters 67, 386 (1991).

${ }^{14}$ M. W. Coffey and J. R. Clem, Physical Review B 45, 9872 (1992)

To C. J. van der Beek, V. B. Geshkenbein, and V. M. Vinokur, Physical Review B 48, 3393 (1993).

16 F. Gömöry, Superconductor Science and Technology 10, 523 (1997).

17 R. Prozorov, R. W. Giannetta, N. Kameda, T. Tamegai, J. A. Schlueter, and P. Fournier, Physical Review B 67, 184501 (2003).

18 C. P. Bean, Physical Review Letters 8, 250 (1962).

19 P. Bak, C. Tang, and K. Wiesenfeld, Physical Review Letters 59, 381 (1987).

${ }^{20}$ H. Kim, N. H. Sung, B. K. Cho, M. A. Tanatar, and R. Prozorov, Physical Review B 87, 094515 (2013).

21 A. I. Larkin and Y. N. Ovchinnikov, Journal of Low Temperature Physics 34, 409 (1979).

22 G. Blatter, V. B. Geshkenbein, and J. A. G. Koopmann, Physical Review Letters 92, 067009 (2004).

23 M. Timmermans, T. Samuely, B. Raes, J. V. de Vondel, and V. V. Moshchalkov, ACS Nano 8, 2782 (2014).

${ }^{24}$ L. Embon, Y. Anahory, A. Suhov, D. Halbertal, J. Cuppens, A. Yakovenko, A. Uri, Y. Myasoedov, M. L. Rappaport, M. E. Huber, A. Gurevich, and E. Zeldov, Scientific Reports 5, 7598 (2015).

25 R. Willa, V. B. Geshkenbein, R. Prozorov, and G. Blatter, Physical Review Letters 115, 207001 (2015).

26 R. Willa, V. B. Geshkenbein, and G. Blatter, Physical Review B 92, 134501 (2015).

27 R. Labusch, Crystal Lattice Defects 1, 1 (1969).

28 E. Zeldov, A. I. Larkin, V. B. Geshkenbein, M. Konczykowski, D. Majer, B. Khaykovich, V. M. Vinokur, and H. Shtrikman, Physical Review Letters 73, 1428 (1994).
29 R. Willa, V. B. Geshkenbein, and G. Blatter, Physical Review B 89, 104514 (2014).

${ }^{30}$ G. Blatter, M. V. Feigel'man, V. B. Geshkenbein, A. I. Larkin, and V. M. Vinokur, Review of Modern Physics 66, 1125 (1994).

31 E. H. Brandt, Journal of Low Temperature Physics 26, 709 (1977).

32 E. H. Brandt, Journal of Low Temperature Physics 26, 735 (1977).

33 G. Blatter and V. B. Geshkenbein, in Superconductivity, edited by K. Bennemann and J. Ketterson (Springer Berlin Heidelberg, 2008) pp. 495-637.

34 J. A. G. Koopmann, V. B. Geshkenbein, and G. Blatter, Physica C 404, 209 (2004).

35 Y. N. Ovchinnikov and B. I. Ivlev, Physical Review B 43, 8024 (1991).

36 A. Schönenberger, A. I. Larkin, E. Heeb, V. B. Geshkenbein, and G. Blatter, Physical Review Letters 77, 4636 (1996).

37 A. E. Koshelev, private communication.

38 E. H. Brandt, Physical Review B 34, 6514 (1986).

39 V. L. Ginzburg and L. D. Landau, [L. D. Landau, Collected papers p. 546 (1965), Pergamon Press, Oxford] Zh. Eksp. Teor. Fiz. 20, 1064 (1950).

40 A. Schmid, Physik der kondensierten Materie 5, 302 (1966).

41 J. R. Clem, Journal of Low Temperature Physics 18, 427 (1975).

42 D. Saint-James, G. Sarma, and E. J. Thomas, Type II Superconductivity, International series of monographs in natural philosophy, Vol. 17 (Oxford: Pergamon, 1969).

43 G. J. Ruggeri and D. J. Thouless, Journal of Physics F: Metal Physics 6, 2063 (1976).

44 P. G. DeGennes, Superconductivity of Metals and Alloys (West View Press, 1966).

45 S. H. Autler, E. S. Rosenblum, and K. H. Gooen, Physical Review Letters 9, 489 (1962).

46 W. DeSorbo, Review of Modern Physics 36, 90 (1964).

47 L. P. Gorkov, [Zh. Eksp. Teor. Fiz. 36, 1918 (1959)] JETP 9, 1364 (1959).

48 J. Lowell, Journal of Physics F: Metal Physics 2, 559 (1972).

49 R. T. Gordon, N. D. Zhigadlo, S. Weyeneth, S. Katrych, and R. Prozorov, Physical Review B 87, 094520 (2013).

50 N. H. Sung, Y. J. Jo, and B. K. Cho, Superconductor Science and Technology 25, 075002 (2012).

51 D. Roditchev, C. Brun, L. Serrier-Garcia, J. C. Cuevas, V. H. L. Bessa, M. V. Milosevic, F. Debontridder, V. Stolyarov, and T. Cren, Nature Physics 11, 332 (2015). 